\title{
The Interplay of Aesthetics, Usability and Credibility in Mobile Website Design and the Effect of Gender
}

\author{
Kiemute Oyibo and Julita Vassileva \\ Computer Science \\ University of Saskatchewan \\ Saskatoon, S7N 5C9, Canada \\ kiemute.oyibo@usask.ca,jiv@cs.usask.ca
}

\begin{abstract}
In human-computer interaction, aesthetics, usability and credibility are key factors in the design of a successful website. Specifically, aesthetics has been identified as one of the main drivers of web credibility. However, in the mobile domain, large-scale research, cutting across cultures and continents, which is key to the generalizability of findings, is scarce. To bridge this gap, we conducted a multicultural study among 526 participants from 5 continents: Africa, Asia, North America, South America and Europe. Using four systematically designed mobile websites, we investigated: (1) the interrelationships among aesthetics, usability and credibility; and (2) the moderating effect of gender. Our results, based on partial least square path modeling, reveal that: (1) perceived aesthetics is stronger than perceived usability in predicting the perceived credibility of mobile websites; and (2) gender moderates the effect of perceived aesthetics on perceived usability, with this effect being stronger for males than for females. Our findings underscore the need for designers to pay closer attention to aesthetics in designing successful mobile websites, as their visual appeal, irrespective of gender, enhances their perceived ease of use and credibility. These findings are noteworthy because, given the usability challenges posed by the relatively small-screen size of the mobile device, designers may be tempted to focus on designing easy-to-use websites only, while downplaying their visual appeal. Such a decision may adversely impact the overall credibility of their websites going by users' first impression.
\end{abstract}

Keywords-mobile website; path model; aesthetics; usability; credibility; gender, user inteface, design layout, perception

\section{INTRODUCTION}

The perceived credibility of a website is critical to the commercial success of its owners [1]. According to Fogg [2], "those who can design for credibility gain a strategic advantage" (p. 10) in the marketplace. Web credibility has been identified as an important factor in web design since it is the first point of contact with a business for new customers. As such, it is one of the most valuable assets of an organization. According to Fogg [2], credibility gives designers the power to change both the attitudes and behaviors of users. The high credibility of a website can foster positive user attitudes, such as thinking favorably of the website owners, feeling comfortable interacting with the website and embracing the point of view of the website owners. Similarly, a site's credibility can influence users' behaviors, for example, registering with the website by providing their personal information, completing purchases of products and services and returning to the site some other day [3]. Moreover, research [4]-[7] has shown that aesthetics and usability are key factors in determining the perceived credibility of and intention to use websites. While a lot of research has been carried out on the influence of aesthetics and of usability [8] on credibility [1], [9], it remains to be seen in the mobile domain how these influences play out with respect to a mixed population comprised of different cultures, and how gender moderates the interrelationships among these three web design constructs [10]. Investigating this research topic has become important given: (1) the debate [11]-[16] regarding which of aesthetics (a hedonic attribute) and usability (an instrumental attribute) is more important, and thus should be given priority, in humancomputer interaction (HCI) and user interface (UI) design; and (2) the temptation for designers to focus more on usability and less on aesthetics owing to the usability challenges the relatively small-screen size of the mobile device poses in human-computer interaction [17], [18].

In HCI, aesthetics and usability can be described as two key factors among a broad range of factors that influence the user experience. Morville [19] identified seven factors, which make up the User Experience Honeycomb. They include usefulness, usability, findability, credibility, desirability, accessibility and value. Of these factors, desirability (which relates to aesthetics), usability and credibility are among the most researched design constructs in the literature [1], [8], [9], [15], [20]-[23]. In particular, there have been many debates [12], [13], [22], [24], [25] between usability experts and graphics designers regarding which of aesthetics and usability is more important to users in judging a website based on their initial experience [26]. While some researchers and practitioners [24], [27] have argued that usability (a utilitarian factor) is very important in the design of HCI artifacts, others [1], [9], [22], [28] have stressed the need to pay attention to aesthetics (a hedonic factor) as well, as it is the first port of call in judging a website and the predominant determinant of web credibility. As argued by the Interaction Design Foundation [19], it is true that user experience as a discipline started with usability; however, it has outgrown usability to include other important factors, which designers must accommodate in order to deliver more effective and successful applications to their target users. Consequently, aesthetics has been identified as one of these factors, which is an important aspect of the desirability of a product, in general [19]. 
Furthermore, aesthetics has been identified as a key factor in the formation of users' first impression about a website, which is made within the first few seconds of encountering a website [29]. First impression is regarded as a key criterion in users' initial judgment of a website because it has to do with feelings and opinions quickly formed unconsciously on the basis of nothing other than perceived aesthetics [30]. According to Reinecke et al. [29], first impression is influential to the extent that it can later affect users' "opinions of a site's usability and trustworthiness" (p. 2049). According to Lidwell, Holden and Butler [31], "aesthetic designs look easier to use and have probability of being used" (p. 18). In general, aesthetic designs have been found to be more effective than less-aesthetic designs (which actually may be more usable) in fostering favorable user attitudes, including more tolerance of design problems [31]. Finally, research [32]-[34] has shown that gender differences exist in the evaluation of websites. Females are generally referred to as comprehensive information processors that pay attention to details, while males are referred to as selective information processors that miss subtle cues [35]. In HCI, females have been found to be driven by intrinsic factors more than males, while males by extrinsic factors more than females [36], [37].

However, most of the above findings regarding aesthetics, usability and credibility, on one hand, and the influence of gender, on the other hand, have been based on: (1) homogeneous samples; and (2) mostly studies (e.g., [10], [38][40]) conducted among the Western and Asian populations. These may threaten generalizability to a multicultural population sample. Moreover, there is limited research on how males and females differ [10], especially with respect to a multicultural population sample. To bridge these gaps, we conducted a large-scale empirical study among 526 subjects from five continents (Africa, Asia, North America, South America and Europe) to investigate the interrelationships among the three web design constructs and the influence of gender, using four systematically designed mobile websites as a case study. The results of our path modeling reveals that perceived aesthetics is stronger than perceived usability in determining the perceived credibility of mobile websites. Our results also reveal that there is a stronger aesthetic-usability effect for males than for females, indicating that males are more affected by the halo-effect phenomenon: a psychological cognitive bias in which the perception of one attribute of an object distorts the perception of other attributes [41], [42].

The rest of the paper is organized as follows. Sections II and III focus on the background and related work, respectively. Section IV and V focus on the research method and results, respectively. Finally, Sections VI, VII and VIII dwell on the discussion, limitation/future work and conclusion, respectively.

\section{BACKGROUND}

In this section, we provide an overview on the three web design constructs investigated in this paper.

\section{A. Credibility}

Credibility is defined as believability [4]. According to Fogg [43], there are four types of web credibility: presumed, surface, reputed, and earned credibility. On one hand, presumed credibility is based on users' general assumptions, while surface credibility is based on their first impressions. On the other hand, reputed credibility is based on third-party endorsements/referrals, while earned credibility is based on a users' firsthand experience, consistent over time [43]. In this paper, we are concerned about presumed/surface credibility, which we refer to as perceived credibility. According to Fogg et al. [4], the perceived credibility of a website is composed of two main dimensions: the perceived trustworthiness of the owners and the perceived expertise of the designers. Robins and Holmes [1] observed that the judgment of the credibility of a website and its content places a burden on the user, since, unlike most journal publications, website information is not subjected to peer reviews or editorial processes. Hence, users adopt visceral means to base their assessment of credibility. Fogg et al. [4], postulated that, in evaluating the credibility of a website, users base their judgment on the assessment of perceived trustworthiness and perceived expertise. They explained that credibility is perceived by the users and does not reside in the evaluated object. In other words, credibility depends on the judgment users make about the object under evaluation. According to the prominence-interpretation theory, postulated by Fogg [44], users assess online credibility based on specific elements they notice in the object under evaluation (prominence of design elements) and the judgment they make about the observed elements (the interpretation they gave them) of the object. He went further to explain that, if a specific element in a website is not noticed by users, it will have no impact on their overall credibility judgment of the site. He identified five factors which may affect the prominence of a website element: user involvement with the website, topic of the website, task of the user, experience of the user and individual differences (ranging from the need for cognition to level of literacy). On the other hand, he identified assumptions in the user's mind (determined by culture, past experience, heuristics, etc.); skill/knowledge (determined by the user's level of competency on the website's subject matter); and context (e.g., the user's environment, expectations, situational norms, etc.) as the main factors that may influence the interpretation of the elements of a website under evaluation [44].

\section{B. Aesthetics}

Aesthetics is related to the notion of beauty. Historically, beauty is regarded as one of the ultimate values in Western philosophy, just like goodness, truth and justice. The nature of beauty has been one of the most fundamental and controversial subjects in philosophical aesthetics, which has endured several ages. It is a central theme among ancient Greek, Hellenistic and medieval philosophers as well as eighteenth and nineteenth century philosophers, such as Shaftesbury, Hutcheson, Kant, Hume and Hegel. Although, in the beginning of the twentieth century, the study of beauty as a subject of philosophical inquiry and as a primary goal of the art witnessed a decline, a new wave of interest began gaining traction in the early years of the twenty-first century [45]. In particular, Baumgarten reappraised the concept of aesthetics, which, prior to the mideighteenth century, had meant "sensibility" ("responsiveness to the stimulation of the senses") [46], [47]. He redefined it as the judgment of taste or that which is beautiful. He based the 
judgment of taste or beauty on the feeling of pleasure or displeasure. Hence, aesthetics is defined as the branch of philosophy, which is concerned with "the nature and appreciation of art, beauty and good taste" [48]. It is derived from the Greek word "aisthetikos", which means "of sense perception" [48]. Moreover, it is part of axiology, which is the study of value judgment. According to [48], the judgment of aesthetic value relies on our ability to discriminate at the sensory, emotional and intellectual levels. However, while Baumgarten referred to the judgment of beauty as cognitive, which has to do with rational ideas, such as harmony, Kant referred to it as affective, which has to do with the form of the object under evaluation [49], [50]. Consequently, Kant emphasizes that "aesthetics judgment must concern itself only with form (shape, arrangement, rhythm, etc.) in the object presented, not with sensible content (color, tone, etc.)" [49].

Furthermore, according to Kant, the aesthetic judgment of an object is a subjective experience, which is disinterested, universal and necessary. It is disinterested in the sense that it is desire-free, meaning we feel or take pleasure in an object because we judge it beautiful, and not the other way round, as held by Baumgarten. On the other hand, it is universal and necessary because the beauty of an object is self-evident and demands others agree with us on its being beautiful. However, according to Kant, although beauty appears as if it were an actual property of the object, it possesses no objective property that makes the object under evaluation beautiful. Rather, the universality and necessity (self-evidence) of aesthetic judgment are a product of the features of the human mind, which Kant called "common sense" [49], [51].

In the context of HCI design, aesthetics is defined as the visual or aesthetic appeal of an artifact (e.g., a website) [52]. Though, in general, there are many forms of aesthetics (e.g., "literary aesthetics"), in this paper, we are particularly concerned with "visual aesthetics" as it relates to web design and the visual sense of perception of users. Thus, we also refer to visual aesthetics as "visual appeal" and/or "perceived aesthetics" from the users' point of view, just like perceived usability and perceived credibility. Though, historically, aesthetics is associated with order and form, in more recent literature, it is associated with delight and perception [21]. More specifically, perceived aesthetics in HCI research is related to the notion of aesthetic judgment, which we covered in the foregoing paragraph.

Based on Lavie and Tractinsky's [21] psychometric analyses on developing a scale for HCI research, it was found that perceived aesthetics is composed of two dimensions: classical and expressive aesthetics. Classical aesthetics pertains to the traditional notion of orderliness and cleanness of the design of an artifact, while expressive aesthetics reflects the originality and creative power of the designer of the artifact. In $\mathrm{HCI}$ and web design, classical aesthetics is operationalized by the use of simple terms such as "visual," "pleasant," "clean," etc., while expressive aesthetics is operationalized by the use of complex terms such as "fascinating," "creative," "sophisticated," etc. [21], [53], [54]. Furthermore, perceived aesthetics may depend on a number of factors, such as gender, culture, etc. Thus, for example, what one culture/gender may perceive as aesthetic may be perceived as unaesthetic by another culture/gender [42].

\section{Usability}

Usability is defined as the ease of use of an information system [55]. In other words, it captures how effective and free of efforts humans can interact with an information system (e.g., website) [56]. Basically, we have two types of usability: perceived and actual usability [13]. Empirically, perceived usability can be measured by asking users to give their opinions about their perceived ease of use of a system. Thus, it can be operationalized using terms such as "easy to use", "easy to navigate", "convenient to use", etc. [21]. On the other hand, actual usability can be measured objectively by recording how well users can master and perform tasks using a system [56].

\section{RELATED WORK}

A number of studies, empirical and experimental, have been carried out to investigate the interrelationships among aesthetics, usability and credibility ("perceived" omitted from the names of the constructs, for brevity, here and other parts of the paper). In this section, we review related works in this area.

\section{A. Aesthetics-Usability Relationship}

There have been a lot of studies on the relationship between aesthetics and usability (aka "aesthetic-usability effect" [31]). Kurosu and Kashimura [28] conducted an experimental study among 252 students in Japan to investigate the determinants of the perceived usability, which they called apparent usability, of an information system. The study was based on an Automated Teller Machine (ATM), which had 26 different layouts. Their correlation analysis revealed that the apparent usability of the ATM was more strongly impacted by its perceived aesthetics than by its inherent usability. Thus, they concluded that perceived aesthetics influences perceived usability more than inherent usability does. As a follow-up, Tractinsky [22] conducted a similar study among 104 first-year engineering students in Israel, using the same study materials (26 ATM layouts translated into Hebrew from Japanese). The study was carried out in an attempt to replicate Kurosu and Kashimura's [28] study and uncover the effect of culture. Tractinsky [22], just like Kurosu and Kashimura [28], found a strong correlation between perceived aesthetics and perceived usability, suggesting that the aesthetic-usability effect holds, irrespective of culture. Other studies (e.g., [13], [15], [23], [57]) have also confirmed the relationship between aesthetics and usability. For example, in a further follow-up experimental study, Tractinsky, Katz and Ikar [13] investigated the determinants of the perceived usability of nine of the 26 adapted ATM layouts after users had actually interacted with them. They found that the perceived usability of the ATM after use was influenced mainly by its perceived aesthetics before use and not by its actual usability. Thus, they concluded "what is beautiful is usable." They attributed this finding to the "halo effect" [41], [42]. However, Hassenzahl [58], who questioned this finding, upon attempting to confirm it in a similar experimental study, reported that he was unable to replicate the halo-effect phenomenon. Further, in the mobile domain, Li and Yeh [59], carried out a study on mobile trust using e-commerce websites 
and 200 Taiwanese subjects as a case study. Just as in earlier research [13], [28] in the non-mobile domain, they found that design aesthetics significantly impacts perceived ease of use. Finally, in mobile website design, Oyibo and Vassileva [23] investigated the relationship between classical aesthetics vs. expressive aesthetics and perceived usability. They found that, regardless of culture, classical aesthetics has a stronger influence than expressive aesthetics on perceived usability.

\section{B. Aesthetics-Credibility Relationship}

A number of studies have also been carried out on the link between aesthetics and credibility. Robins and Holmes [1] conducted a study of 42 websites among 20 Information Science students from Kent State University in the United States. They found that when the same information content is presented to users at different levels of aesthetic treatment, the contents with better visual presentation is likely to be perceived as having higher credibility. In the mobile domain, Oyibo, Ali and Vassileva [60] replicated this finding in a mixed-method study of mobile websites with different levels of aesthetic appeal among 285 participants from Africa, North America and Asia. Further, Fogg et al. [9] evaluated the credibility of two actual (health) websites in a qualitative study among over 2500 subjects from around the world, $71 \%$ of whom were from the United States. They found that design look (which is related to perceived aesthetics) is the primary determinant of the perceived credibility of the investigated websites. Similarly, Liu, Lee and Lee [6] conducted a study among 65 junior and sophomore students from the Department of Digital Media Design in a Taiwanese university on the connection between perceived aesthetics (which they referred to as appearance) and perceived credibility. Their study was based on the empirical evaluation of a number of online banking websites. They found that better-looking websites were rated as more credible than less-good-looking ones. Further, in their investigation of mobile websites using partial least square path modeling and Canada and Nigeria as a case study, Oyibo and Vassileva [7] found a strong relationship between perceived aesthetics and perceived credibility.

\section{Usability-Credibility Relationship}

A number of studies have investigated the relationship between usability and credibility in web design. Fogg et al. [4] conducted a study of 51 websites among over 1400 participants from the Unites States and Europe to determine the factors that drive the perceived credibility of websites. They found ease of use (coming behind real world feel) as the second strongest determinant of perceived credibility. $\mathrm{Li}$ and $\mathrm{Yeh}$ [61] also conducted a study among 200 Taiwanese on mobile commerce websites. They found a significant relationship between perceived ease of use and mobile trust, which is a component of mobile credibility [4]. Youngblood and Mackiewicz [16] conducted a usability evaluation of government websites in the United States. They found that perceived usability impacts the credibility of the investigated websites. Similarly, Oyibo and Vassileva [62] conducted a study among 233 participants from Canada and Nigeria on mobile web credibility. They found a significant relationship between perceived usability and perceived credibility, which cuts across culture. However, in Neumark et al.'s [63] investigation on the quality and impact of online health information on users, using 29 Hebrew websites as a case study, they found no correlation between the perceived usability and the perceived credibility of the investigated websites.

\section{Effect of Gender in Web Design and Credibility}

Limited research has been done on the impact of gender on the judgment of websites in the mobile domain. In the desktop domain, Cyr [10] conducted a study on website design and gender difference across eight different cultures (Canada, United States, India, Germany, Japan, Mexico, Chile, and China) among 1156 participants. Similarly, in the mobile domain, Oyibo and Vassileva [60] conducted a study among 285 participants from North America, Africa and Asia. While both groups of authors found that females are more critical than males in evaluating websites, they did not investigate how gender moderates the interrelationships among the three web design constructs: aesthetics, usability and credibility, which we are currently investigating in this paper. In fact, most prior studies (e.g., [32], [33], [64]-[66]) in the literature often focus on how both genders differ in terms of participants' preferences and the levels of perception of certain design attributes - and neither in terms of the interrelationships among the three web design constructs nor the influence of one design construct on another in a partial least square path model or structural equation model, where constructs compete against one another in terms of magnitude of influence or effect size. Moreover, most prior studies in the extant literature have been:

1. concentrated on subjects mainly from Western and Asian cultures (e.g., [6], [9], [10], [28]);

2. based on monocultural and convenience samples, involving mainly student subjects (e.g., [1], [6], [22], [28]); and

3. focused on the desktop domain (e.g., [1], [16], [64], [66]).

These limitations may threaten the generalizability of findings to the mobile domain and a mixed population sample, which cuts across different cultures and geographical locations. Our study attempts to bridge these gaps by using a more globally diverse population sample, which includes subjects from different cultures and continents, including understudied continents such as Africa and South America.

\section{METHOD}

This section covers our research question, research design, measurement instruments and participants' demographics.

\section{A. Research Questions}

In this paper on the perception and evaluation of mobile web design, using a multicultural population sample, we aim to answer the following two main research questions:

1. How do the three important web design constructs (aesthetics, usability and credibility) influence one another in the mobile web design?

2. Do the interrelationships among aesthetics, usability and credibility depend on gender and/or UI design? 


\section{B. Research Design}

To answer our research questions, we systematically designed four versions of a hypothetical mobile website ${ }^{1}$ based on UI designs adapted from the marketplace (see Fig. 1 [67]). The websites comprise two basic types of designs: lowaesthetic design websites (WA and WB) and high-aesthetic design websites (WC and WD). We arrived at these four websites using a systematic transformation framework we called artifact-action framework. The quadrants of the framework represent the artifacts (web designs), while the axes represent the actions (manipulations) carried out on one web design (in a given quadrant) to arrive, in a clockwise fashion, at a new web design (in the next quadrant). For example, starting from WA (a multi-color design), to arrive at WB (a minimalistic design), we carry out the action "make gray and add icon." Further, to arrive at WC (a blue-theme design) from WB, we carry out the action "make unicolor." This continues in a clockwise manner until we arrive at the initial web design (WA) we started from.

\section{Research Hypotheses}

Based on the path model in Fig. 2, we formulated eight hypotheses, relating to the four UIs in Fig. 1, as follows:

H1: The direct effect of Aesthetics (A) on Credibility $(C)$ is stronger than the direct effect of Usability $(U)$ on Credibility $(C)$.

H2: The direct effect of Aesthetics on Usability is stronger for the female group than for the male group.

H3: The direct effect of Aesthetics on Credibility is stronger for the female group than for the male group.

H4: The effect size of Aesthetics on Credibility is stronger for the female group than for the male group.

H5: The direct effect of Usability on Credibility is higher for the male group than for the female group.

H6: The effect size of Usability on Credibility is higher for the male group than for the female group.

H7: The mediation of the direct effect of Aesthetics on Credibility by Usability is higher for the grid-layout mobile design than the list-layout mobile designs.

H8: The mediation of the direct effect of Aesthetics on Credibility by Usability is stronger for the male group than for the female group.

We used a within-subject design, with all four mobile webpages (UIs) presented to each participant in this orderWC, WA, WB and WD-in the online survey. To minimize the possible impact of the presentation order, we specifically ensured that the mobile webpages were not presented (based on

${ }^{1}$ We adapted all four websites from existing tourism and travels' mobile websites (m.wakanow.com, mobile.united.com, mobile.utah.com and tourismwinnipeg.com) in the market in 2014. However, some of the websites' UIs must have been redesigned [72].

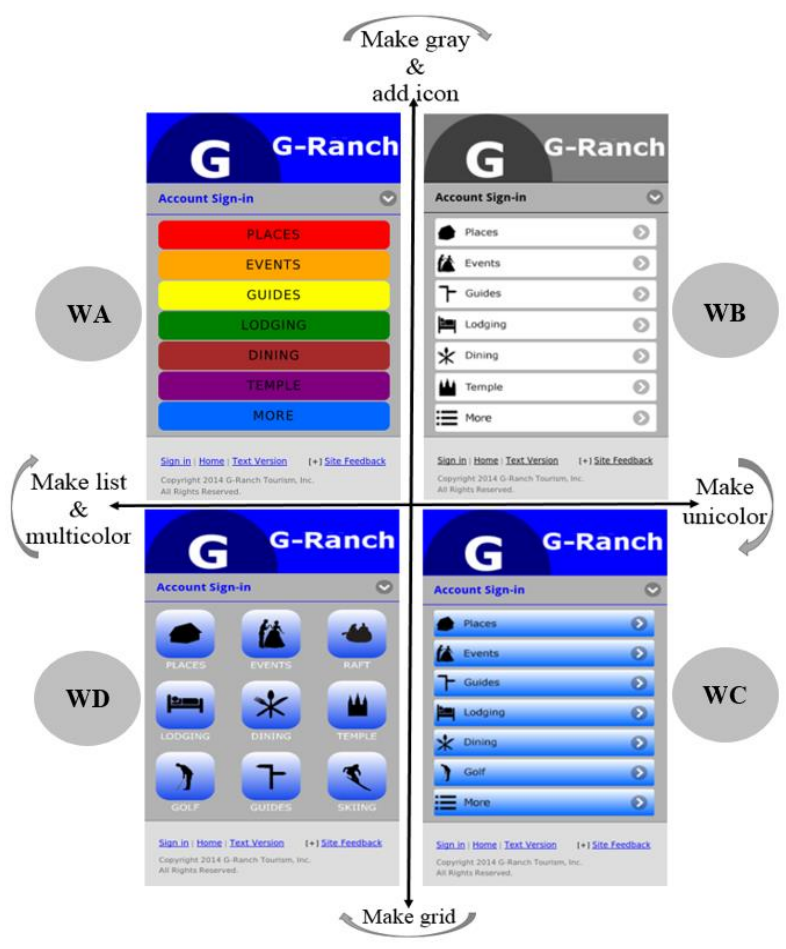

Fig. 1. Systematically designed user interfaces

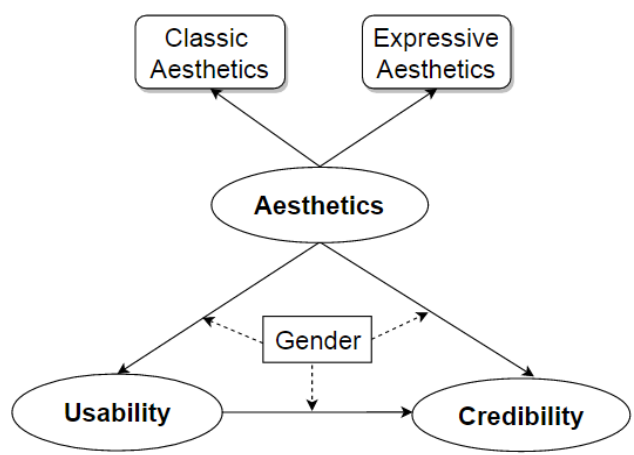

Fig. 2. Hypothesized path model.

our perception and evaluation) from the most aesthetic to the least aesthetic or vice versa, e.g., starting with WD and WC and ending with WB and WA, or vice versa.

The hypothetical model in Fig. 2, as well as the eight hypotheses above, are informed by prior findings in the literature. The first hypothesis (H1) was informed by the findings by Robins and Holmes [1], Fogg et al. [9] and other researchers [7], [15] in the desktop domain, with respect to web design and credibility. The authors, in different studies, found that perceived aesthetics is the predominant design attribute that determines the credibility of a website. For example, according to Robins and Holmes [1], aesthetics is the main factor that may influence a user to either stay on a website upon the first visit or move to another. The second to the sixth hypotheses ( $\mathrm{H} 2$ to $\mathrm{H} 6$ ) were based on the assumption 
that if a given gender is more concerned about any of the two exogenous constructs (aesthetics or usability) than the other gender, then the influence of that exogenous construct on the endogenous constructs (usability or credibility) will be stronger for that gender than the other. Generally, research has shown that men are more motivated by extrinsic factors (e.g. usability), which are task-oriented, than women, while women, on the other hand, are more motivated by intrinsic factors, which are hedonic in nature (e.g., aesthetics), than men [68]. Thus, with respect to $\mathrm{H} 2, \mathrm{H} 3$ and $\mathrm{H} 4$, we hypothesized that the influence and effect size of aesthetics on credibility ( $\mathrm{H} 3$ and $\mathrm{H} 4$, respectively), on one hand, and the influence of aesthetics on usability ( $\mathrm{H} 2)$, on the other hand, will be stronger for females than for males because prior research [7], [69], [70] has shown that females care about aesthetics more than males do. As such, we hypothesized that aesthetics may have more effect on the judgment of women than men [71]. With respect to $\mathrm{H} 5$ and H6, we hypothesized that the influence and effect size of usability on credibility will be stronger for males than females because prior research (e.g., [60], [69]) has shown that males care about usability more than females.

Furthermore, the seventh hypothesis (H7) was based on the fact that mobile WD possesses a unique layout, which is different from the other UIs. Thus, we hypothesized that the grid layout (having more spaced-out items, which makes it more usable or less prone to click-errors than the others [72]) will result in perceived usability influencing perceived credibility more for mobile WD than for the other mobile webpages, which has a list layout (having less spaced-out items, which may cause more click-errors). Thus, as stated in the seventh hypothesis ( $\mathrm{H} 7)$, we expected to have usability mediating the influence of aesthetics on credibility more for mobile WD than for mobile WA, WB and WC. Finally, the eighth hypothesis (H8) is formulated based on the same assumption made regarding the fifth hypothesis (H5). Basically, our eighth hypothesis (H8) is that, if the influence of usability on credibility is stronger for the male group than the female group, then, usability will mediate the influence of aesthetics on credibility for the male group more than for the female group.

\section{Measurement Instruments}

To measure all three web design constructs in our hypothesized path model, we used existing scales that have been previously validated. To measure perceived aesthetics, we used the six-item scale of Lavie and Tractinsky [5] as adapted by van Schaik and Ling [73]. The aesthetics scale comprises two subscales: classical aesthetics and expressive aesthetics. Similarly, to measure perceived usability, we used Lavie and Tractinsky's five-item scale [5] as adapted by De Angeli, Sutcliffe and Hartmann [74]. Finally, to measure perceived credibility, we used a single-item scale [75], which has been shown by prior research [76], [77] to be as reliable as a multiitem scale. Each item in each of the scales was measured using a 7-point Likert scale, ranging from "Strongly Disagree (1)" to "Strongly Agree (7)." In administering the online survey, all of the items from both aesthetics and usability scales for each mobile webpage were presented together in a randomized fashion to each participant. This was done with the intention to prevent participants from easily identifying the design construct we are measuring at a given instance in time, e.g., if we presented each construct's items separately in different blocks under the image of each webpage [23]. The credibility question was asked immediately after the randomized questions on aesthetics and usability had been asked.

\section{E. Participants}

Our study was submitted to our university's research ethics board for evaluation and approval. Thereafter, upon approval, we invited and recruited participants, using our university's website, Facebook and email, to take part anonymously in our online survey. In order to appreciate them for their time, participants were given an opportunity to optionally enter for a draw for a chance to win one of our four gift cards of C\$50 each. About 550 participants took part in the study as a whole. However, after cleaning the data, we were left with a total of 526 participants for our final analysis. Table I shows the demographic information of the participants, which comprised $53.6 \%$ males and $44.5 \%$ females.

TABLE I. PARTICPANTS' DEMOGRAPHICS ACCORDING TO COUNTRIES.

\begin{tabular}{|c|c|c|c|c|c|c|c|c|c|}
\hline Criterion & Group & Nigeria & Ghana & Brazil & China & Canada & Others & Overall & Perc. \\
\hline \multirow{3}{*}{ Gender } & Male & 115 & 37 & 34 & 46 & 25 & 25 & 282 & $53.6 \%$ \\
\hline & Female & 40 & 13 & 16 & 63 & 71 & 31 & 234 & $44.5 \%$ \\
\hline & Unidentified & 1 & 0 & 0 & 6 & 2 & 1 & 10 & $1.9 \%$ \\
\hline \multirow[b]{3}{*}{ Age } & $18-24$ & 118 & 6 & 16 & 51 & 55 & 18 & 264 & $50.2 \%$ \\
\hline & $25-34$ & 32 & 40 & 21 & 59 & 31 & 31 & 214 & $40.7 \%$ \\
\hline & $>34$ & 2 & 3 & 13 & 5 & 12 & 6 & 41 & $7.8 \%$ \\
\hline \multirow{2}{*}{$\begin{array}{l}\text { Years on } \\
\text { Internet }\end{array}$} & $<10$ & 116 & 12 & 8 & 82 & 13 & 9 & 230 & $43.7 \%$ \\
\hline & $>=10$ & 50 & 38 & 42 & 33 & 85 & 48 & 296 & $56.3 \%$ \\
\hline \multirow{6}{*}{$\begin{array}{l}\text { Educational } \\
\text { Qualification }\end{array}$} & Technical/Trade & 8 & 0 & 1 & 12 & 4 & 1 & 26 & $4.9 \%$ \\
\hline & High School & 108 & 2 & 8 & 9 & 40 & 10 & 177 & $33.7 \%$ \\
\hline & Bachelor & 21 & 36 & 13 & 67 & 42 & 13 & 192 & $36.5 \%$ \\
\hline & Postgraduate & 11 & 9 & 28 & 25 & 11 & 31 & 115 & $21.9 \%$ \\
\hline & Subtotal & 156 & 50 & 50 & 115 & 98 & 57 & 526 & $100 \%$ \\
\hline & National Percent & $29.7 \%$ & $9.5 \%$ & $9.5 \%$ & $21.9 \%$ & $18.6 \%$ & $10.8 \%$ & $100 \%$ & \\
\hline
\end{tabular}




\section{RESULT}

In this section, we present the results from our Partial Least Square Path Modeling (PLSPM) using R's plspm package [78].

\section{A. Evaluation of Global Measurement Model}

One of the requirements for the analysis of the structural model is the evaluation of the measurement (inner) model [78].
For the global model, we present the evaluation of the measurement model for all three constructs under investigation (aesthetics, usability and credibility) as shown in Table II.

1) Indicator Reliability: Most of the indicators in the measurement models have an outer loading greater than 0.7 [6]. As such, the reliability criterion is met, as the communality values for most indicators are greater than 0.5 .

TABLE II. INTERNAL CONSISTENCY, INDICATOR RELIABILITY, AND CONSTRUCT VALIDITY.

\begin{tabular}{|c|c|c|c|c|c|c|}
\hline Webpage & Block & Indicator & Loading & Communality & AVE & DG.rho \\
\hline \multirow{14}{*}{ WA } & \multirow[b]{2}{*}{ Aesthetics } & Classical lower order indicator & 0.96 & 0.92 & \multirow[b]{2}{*}{0.91} & \multirow[b]{2}{*}{0.95} \\
\hline & & Expressive lower order indicator & 0.95 & 0.91 & & \\
\hline & \multirow{3}{*}{$\begin{array}{l}\text { Classical } \\
\text { Aesthetics }\end{array}$} & Clean & 0.89 & 0.80 & \multirow{3}{*}{0.81} & \multirow{3}{*}{0.93} \\
\hline & & Pleasant & 0.91 & 0.83 & & \\
\hline & & Visual & 0.89 & 0.79 & & \\
\hline & \multirow{3}{*}{$\begin{array}{l}\text { Expressive } \\
\text { Aesthetics }\end{array}$} & Creative & 0.89 & 0.79 & \multirow{3}{*}{0.75} & \multirow{3}{*}{0.90} \\
\hline & & Fascinating & 0.91 & 0.82 & & \\
\hline & & Sophisticated & 0.80 & 0.64 & & \\
\hline & \multirow{5}{*}{ Usability } & Clear design & 0.81 & 0.66 & \multirow{5}{*}{0.72} & \multirow{5}{*}{0.93} \\
\hline & & Convenient to use & 0.88 & 0.78 & & \\
\hline & & Easy to navigate & 0.87 & 0.77 & & \\
\hline & & Easy orientation & 0.84 & 0.71 & & \\
\hline & & Easy to use & 0.84 & 0.71 & & \\
\hline & Credibility & Credibility level & 1.00 & 1.00 & 1.00 & 1.00 \\
\hline \multirow{14}{*}{ WB } & \multirow[b]{2}{*}{ Aesthetics } & Classical lower order indicator & 0.95 & 0.90 & \multirow[b]{2}{*}{0.89} & \multirow[b]{2}{*}{0.94} \\
\hline & & Expressive lower order indicator & 0.94 & 0.88 & & \\
\hline & & Clean & 0.78 & 0.61 & & \\
\hline & Classical & Pleasant & 0.90 & 0.81 & 0.74 & 0.89 \\
\hline & & Visual & 0.89 & 0.80 & & \\
\hline & & Creative & 0.89 & 0.80 & & \\
\hline & Expressive & Fascinating & 0.89 & 0.80 & 0.71 & 0.88 \\
\hline & Aesthetics & Sophisticated & 0.73 & 0.54 & & \\
\hline & & Clear design & 0.81 & 0.65 & & \\
\hline & & Convenient to use & 0.87 & 0.76 & & \\
\hline & Usability & Easy to navigate & 0.88 & 0.77 & 0.74 & 0.94 \\
\hline & & Easy orientation & 0.86 & 0.74 & & \\
\hline & & Easy to use & 0.88 & 0.78 & & \\
\hline & Credibility & Credibility level & 1.00 & 1.00 & 1.00 & 1.00 \\
\hline & & Classical lower order indicator & 0.96 & 0.91 & & \\
\hline & Aesthetics & Expressive lower order indicator & 0.94 & 0.88 & 0.90 & 0.95 \\
\hline & & Clean & 0.79 & 0.62 & & \\
\hline & Classical & Pleasant & 0.90 & 0.82 & 0.75 & 0.90 \\
\hline & Aesthetics & Visual & 0.91 & 0.82 & & \\
\hline & & Creative & 0.90 & 0.82 & 0.70 & \\
\hline & Expressive & Fascinating & 0.90 & 0.82 & & 0.88 \\
\hline WC & Aesthetics & Sophisticated & 0.69 & 0.47 & & \\
\hline & & Clear design & 0.84 & 0.71 & & \\
\hline & & Convenient to use & 0.85 & 0.72 & & \\
\hline & Usability & Easy to navigate & 0.81 & 0.65 & 0.68 & 0.92 \\
\hline & & Easy orientation & 0.82 & 0.67 & & \\
\hline & & Easy to use & 0.82 & 0.67 & & \\
\hline & Credibility & Credibility level & 1.00 & 1.00 & 1.00 & 1.00 \\
\hline & & Classical lower order indicator & 0.96 & 0.92 & & \\
\hline & Aesthetics & Expressive lower order indicator & 0.94 & 0.88 & 0.90 & 0.95 \\
\hline & & Clean & 0.88 & 0.77 & & \\
\hline & Classical & Pleasant & 0.92 & 0.84 & 0.81 & 0.93 \\
\hline & Aesthetics & Visual & 0.91 & 0.83 & & \\
\hline & & Creative & 0.89 & 0.79 & & \\
\hline & Expressive & Fascinating & 0.88 & 0.77 & 0.70 & 0.87 \\
\hline WD & Aesthetics & Sophisticated & 0.72 & 0.52 & & \\
\hline & & Clear design & 0.87 & 0.76 & & \\
\hline & & Convenient to use & 0.88 & 0.77 & & \\
\hline & Usability & Easy to navigate & 0.89 & 0.79 & 0.79 & 0.95 \\
\hline & & Easy orientation & 0.90 & 0.82 & & \\
\hline & & Easy to use & 0.91 & 0.83 & & \\
\hline & Credibility & Credibility level & 1.00 & 1.00 & 1.00 & 1.00 \\
\hline
\end{tabular}




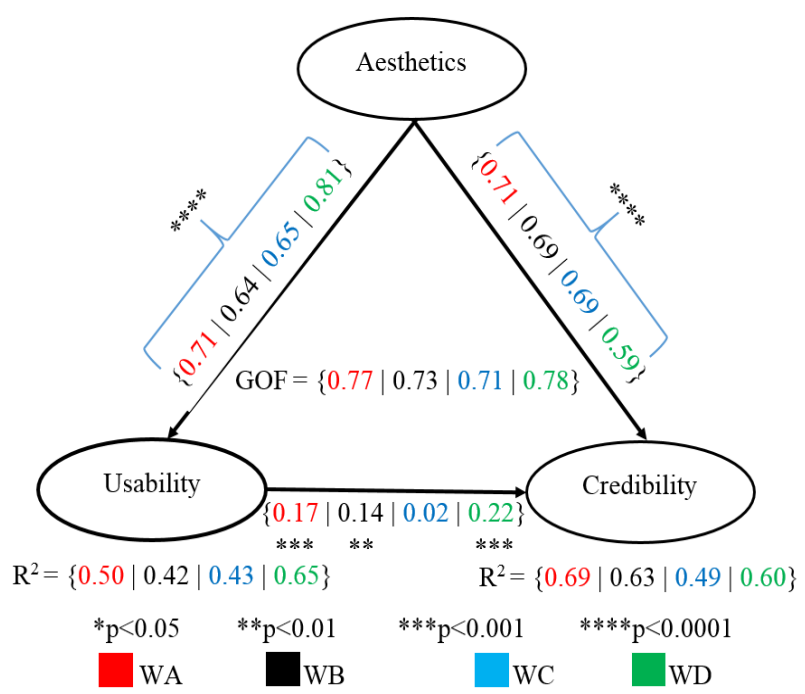

Fig. 3. Global path model.

2) Internal Consistency Reliability: The internal consistency reliability of the constructs was evaluated using the composite reliability criterion, DG.rho $(\rho)$, which is greater than 0.7 for all of the constructs, as shown in in the last column of Table II [79].

3) Convergent Validity: The Average Variance Extracted (AVE) was used to assess convergent validity. The AVE for each model construct was above the recommended value, i.e., greater than 0.5 [79].

4) Discriminant Validity: The crossloading of each construct was also assessed to determine the discriminant validity of each construct. No indicator loaded higher on any other construct than the one it was meant to measure [79].

\section{B. Evalaution of Subgroup Measurment Models}

Our multigroup analysis (MGA) based on gender shows that there is a statistically significant difference between males and females with respect to specific relationships in the global model (see Table III). This led us to build specific models for the male and female subgroups. As such, just as we did for the global model, we evaluated the measurement models for both subgroups. The result showed that both inner models met the required criteria (assessed and presented in the previous subsection) as well. Due to limited space, and for the sake of brevity, the results are not included in the paper.

\section{Analysis of Global Model}

Upon establishing that the measurement models met the required assessment criteria, we carried out an evaluation of the structural models shown in Fig. 3 for all four mobile webpages \{WA | WB | WC |WD\}. The evaluation analysis of the structural models was based on the overall (global) data. For brevity, we have compacted all four global models for all four mobile webpages into one overarching model for quick and easy visualization and side-by-side comparison across all four models.

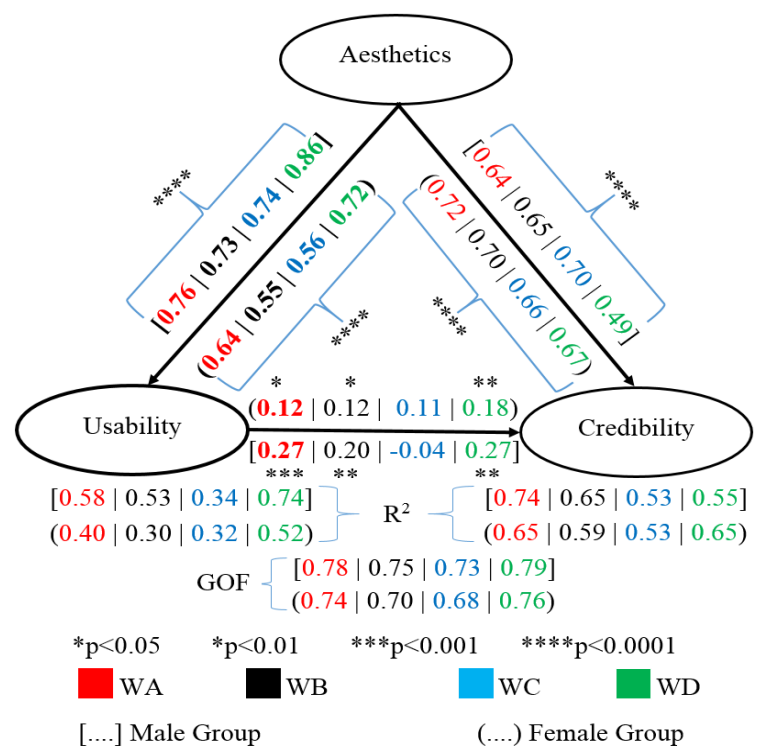

Fig. 4. Subgroup path model for male and female groups (bold fonts indicate paths where there are significant differences between males and females).

The path model shows: (1) the direct effects $(\beta)$ exerted by the exogenous constructs (independent variables) on the endogenous constructs (dependent variables); (2) the coefficients of determination $\left(\mathrm{R}^{2}\right)$ of the endogenous constructs (defined as the amount of variance in an endogenous construct explained by the exogenous constructs); and (3) the goodness of fit (GOF), which indicates the predictive power of each model or how well each model fits its data [78]. The number of asterisks indicates how significant each of the direct effects is, with one, two, three and four asterisks representing the pvalues of $0.05,0.01,0.001$ and 0.0001 , respectively. The pvalue of 0.05 indicates our chosen minimum confidence level ( $\alpha=95 \%$ ) for the direct effect, also known as influence or path coefficient $(\beta)$ in our paper.

Fig. 3 reveals that the global models fit their respective data well due to the high GOF > 0.7 for all four models, with mobile WA and WD having the highest values of 0.77 and 0.78 , respectively, and mobile $\mathrm{WB}$ and $\mathrm{WC}$ having the least values of 0.73 and 0.71 , respectively. Further, based on the PLS-PM standard, $\mathrm{R}^{2}<0.30$ is low, $0.30<\mathrm{R}^{2}<0.50$ is moderate and $\mathrm{R}^{2}>0.60$ is high [78]. Thus, the $\mathrm{R}^{2}$ for usability is moderate for mobile WA, WB and WC (50\%, $42 \%$ and $43 \%$, respectively) but high for mobile WD $(65 \%)$. This indicates that the model accounts for more variance in usability for mobile WD $(65 \%)$ than for the other mobile webpages. For credibility, the $\mathrm{R}^{2}$ values range from $49 \%$ (moderate) for $\mathrm{WC}$ to $69 \%$ (high) for mobile WA, indicating more of the variance in credibility for mobile WA is accounted for than for other webpages. In summary, the global models have high predictive power and explain moderate-to-high amount of the variance in the endogenous constructs (usability and credibility). Further, we see that all the direct effects are significant, except for the direct effect of usability on credibility $\left[(\mathrm{U} \rightarrow \mathrm{C})_{\mathrm{DIR}}\right]$ for mobile WC, with $\beta=0.02$. The path coefficients $(\beta)$ are highly significant for $(A \rightarrow U)_{D I R}$ and $(A \rightarrow C)_{D I R}$ paths (ranging from 
0.59 to 0.81 at $\mathrm{p}<0.0001$ for mobile WD) and quite significant for $(\mathrm{U} \rightarrow \mathrm{C})_{\mathrm{DIR}}$ paths (ranging from 0.14 at $\mathrm{p}<0.01$ for mobile WB to 0.22 at $p<0.001$ for mobile WD). However, there was need for us to find out whether these path coefficients were moderated by gender by carrying out a multigroup analysis.

\section{Multigroup Analysis}

From the multigroup analysis (MGA) we conducted for the male and female subgroups, we found statistically significant differences in the $(\mathrm{A} \rightarrow \mathrm{U})_{\text {DIR }}$ path for all four mobile webpages and in the $(\mathrm{U} \rightarrow \mathrm{C})_{\text {DIR }}$ path for mobile WA only (at $\left.\mathrm{p}<0.05\right)$, as shown in the MGA results in Table III and indicated by the bold fonts in Fig. 4. In all five paths, where significant differences exist, the coefficients are stronger for the male group than for the female group. For example, for the $(\mathrm{A} \rightarrow \mathrm{U})_{\text {DIR }}$ path, the male and female groups have path coefficients $[0.76|0.73| 0.74 \mid 0.86]$ and $(0.64|0.55| 0.56 \mid$ $0.72)$ at $\mathrm{p}<0.0001$ for mobile WA, WB, WC and WD, respectively. Similarly, for the $(\mathrm{U} \rightarrow \mathrm{C})_{\text {DIR }}$ path, the male and female groups have path coefficients of 0.27 at $p<0.001$ and 0.12 at $\mathrm{p}<0.05$, respectively, for mobile WA.

\section{E. Direct, Indirect and Total Effects}

As shown in Fig. 5, we plotted the direct effects $\left[(\mathrm{A} \rightarrow \mathrm{C})_{\mathrm{DIR}}\right.$ and $\left.(\mathrm{U} \rightarrow \mathrm{C})_{\mathrm{DIR}}\right]$, the indirect effects $\left[(\mathrm{A} \rightarrow \mathrm{C})_{\mathrm{IND}}\right]$ and the total effects $\left[(\mathrm{A} \rightarrow \mathrm{C})_{\text {Tот }}\right]$ in order to easily visualize the trend of effects as the web design is systematically manipulated from the least aesthetic design (WA) to the most aesthetic design (WD). (See [67] for the aesthetic ratings.) A remarkable trend in the plot is that the total effect trace $\left[(\mathrm{A} \rightarrow \mathrm{C})_{\text {Tот }}\right]$ for all four webpages is in sync with the direct effect $\left[(\mathrm{U} \rightarrow \mathrm{C})_{\mathrm{DIR}}\right]$ and indirect effect $\left[(\mathrm{A} \rightarrow \mathrm{C})_{\mathrm{IND}}\right]$ traces, with a sharp dip recorded at WC in the global and male models. This reflects the fact that, analytically, the total effect $\left[(\mathrm{A} \rightarrow \mathrm{C})_{\text {TOT }}\right]$ is a function of the direct effect $\left[(\mathrm{A} \rightarrow \mathrm{C})_{\mathrm{DIR}}\right]$ and indirect effect $\left[(\mathrm{A} \rightarrow \mathrm{C})_{\mathrm{IND}}\right]$, while the indirect effect $\left[(\mathrm{A} \rightarrow \mathrm{C})_{\mathrm{IND}}\right]$ is a function of the direct effects $\left[(\mathrm{A} \rightarrow \mathrm{U})_{\mathrm{DIR}}\right.$ and $\left.(\mathrm{U} \rightarrow \mathrm{C})_{\mathrm{DIR}}\right]$. Another interesting finding common to all three plots (in Fig. 5) is that there is a significant indirect effect of aesthetics on credibility via usability for mobile WA, WB and $\mathrm{WD}$, but none for mobile WC. This indirect effect $\left[(A \rightarrow C)_{\text {IND }}\right]$ is relatively stronger in the global and male models. We provide a possible explanation for this finding in the discussion of the eighth hypothesis (H8) in Section VI.

\section{F. Mediation}

We analyzed the global and subgroup models to determine whether usability mediates the effect of aesthetics on credibility. As shown in Fig. 6, for both models, we computed and plotted a measure of mediation, variance accounted for (VAF), which represents the ratio of the significant indirect effect to the total effect on credibility [79]. Based on the recommended guideline [79], which states that $\mathrm{VAF}<0.2$ indicates no mediation, $0.2 \leq \mathrm{VAF} \leq 0.8$ indicates partial mediation and VAF $>0.8$ indicates full mediation, we found that mediation by usability only occurred for: (1) the global model with respect to mobile WD, in which the VAF is 0.23 ; and (2) the male model with respect to mobile WA and WD, in which the VAFs are 0.24 and 0.32 , respectively. It is noteworthy that there is no mediation for: (1) mobile WC because the $(\mathrm{U} \rightarrow \mathrm{C})_{\text {DIR }}$ path coefficient in Fig. 3 and Fig. 4 is non-significant; and (2) mobile WB because VAF $<0.20$, as shown in Fig. 6.

TABLE III. Multigroup ANALysis BETWEEN MALE AND FEMALE

\begin{tabular}{|c|c|c|c|c|c|}
\hline Webpage & Path & Global & M & F & Sig \\
\hline \multirow{4}{*}{ WA } & $(\mathrm{A} \rightarrow \mathrm{U})_{\text {DIR }}$ & 0.71 & 0.76 & 0.64 & 0.05 \\
\cline { 2 - 6 } & $(\mathrm{A} \rightarrow \mathrm{C})_{\text {DIR }}$ & 0.71 & 0.64 & 0.72 & n.s \\
\cline { 2 - 6 } & $(\mathrm{U} \rightarrow \mathrm{C})_{\text {DIR }}$ & 0.17 & 0.27 & 0.12 & 0.05 \\
\hline \multirow{4}{*}{ WB } & $(\mathrm{A} \rightarrow \mathrm{U})_{\text {DIR }}$ & 0.67 & 0.73 & 0.55 & 0.001 \\
\cline { 2 - 6 } & $(\mathrm{A} \rightarrow \mathrm{C})_{\text {DIR }}$ & 0.69 & 0.65 & 0.70 & n.s \\
\cline { 2 - 6 } & $(\mathrm{U} \rightarrow \mathrm{C})_{\text {DIR }}$ & 0.15 & 0.20 & 0.12 & n.s \\
\hline \multirow{3}{*}{ WC } & $(\mathrm{A} \rightarrow \mathrm{U})_{\text {DIR }}$ & 0.65 & 0.74 & 0.56 & 0.001 \\
\cline { 2 - 6 } & $(\mathrm{A} \rightarrow \mathrm{C})_{\text {DIR }}$ & 0.69 & 0.70 & 0.66 & n.s \\
\cline { 2 - 6 } & $(\mathrm{U} \rightarrow \mathrm{C})_{\text {DIR }}$ & 0.02 & -0.04 & 0.11 & n.s \\
\hline \multirow{3}{*}{ WD } & $(\mathrm{A} \rightarrow \mathrm{U})_{\text {DIR }}$ & 0.81 & 0.86 & 0.72 & 0.001 \\
\cline { 2 - 6 } & $(\mathrm{A} \rightarrow \mathrm{C})_{\text {DIR }}$ & 0.59 & 0.49 & 0.67 & n.s \\
\cline { 2 - 6 } & $(\mathrm{U} \rightarrow \mathrm{C})_{\text {DIR }}$ & 0.22 & 0.27 & 0.18 & n.s \\
\hline
\end{tabular}
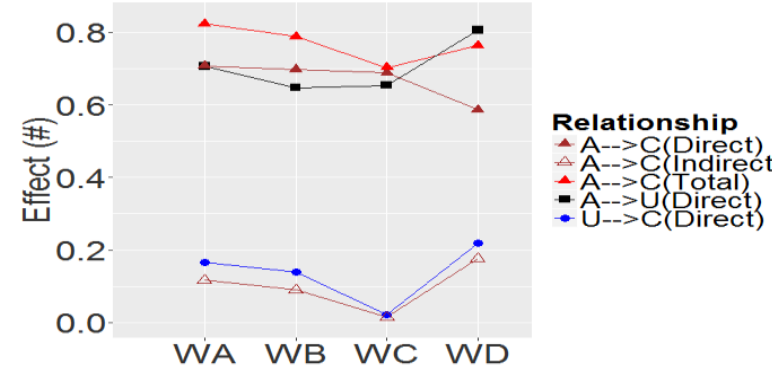
$\triangle A-\rightarrow C$ (nndirect) - A-->U (Diral - A-->C(Direct)

Fig. 5a. Direct, indirect and total effect for the global model

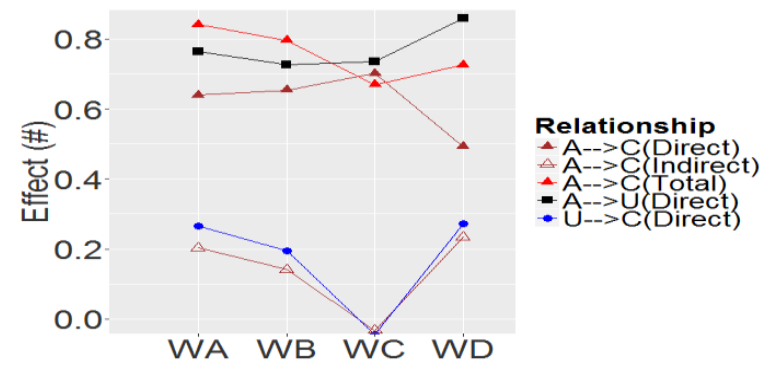

Fig. 5b. Direct, indirect and total effect for the male model

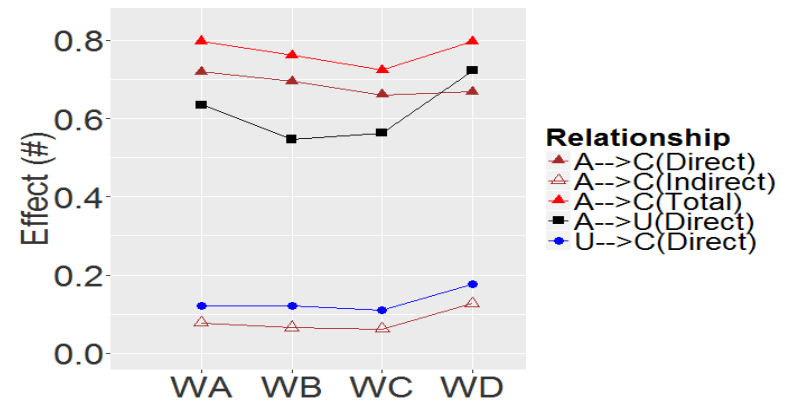

Fig. 5c. Direct, indirect and total effect for the female model 


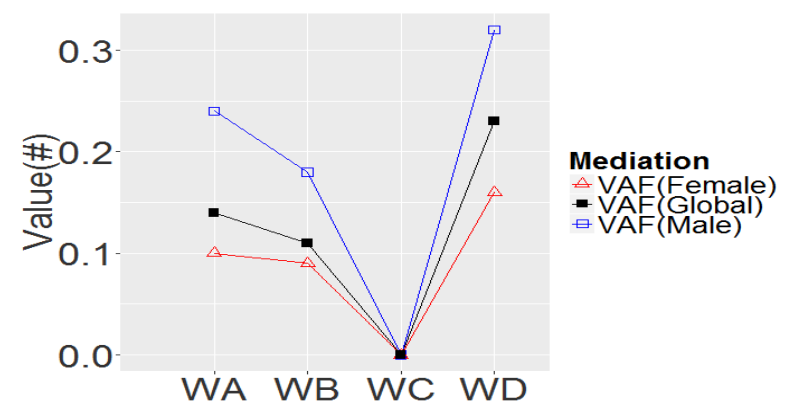

Fig. 6. Usability mediation effect of aesthetics on credibility

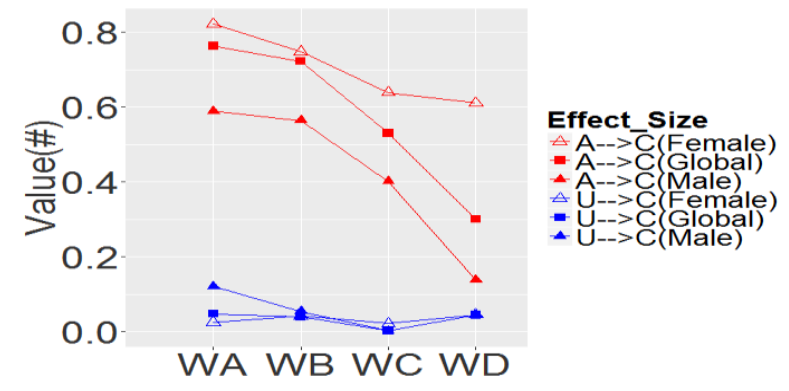

Fig. 7. Effect sizes of driver constructs on credibility

\section{G. Effect Size}

We computed the effect sizes of both exogenous/driver constructs (aesthetics and usability) on the endogenous/target construct (credibility). We used the formula recommended by Hair et al. [79]. According to Preacher and Kelly [80], it has become very important for researchers to report the effect sizes ( $\mathrm{f}^{2}$ or $\mathrm{ES}$ ) of the driver constructs on the target construct whenever possible. Cohen [81] defined effect size as the extent to which the phenomenon of interest is present among the studied population. Effect size could also be defined as a measure of how strong the relationship between two variables of interest is [82]. Fig. 7 shows a line graph of the effect sizes for the driver constructs (aesthetics and usability) on credibility. According to Cohen's guidelines [81], effect sizes of $0.02,0.15$ and 0.35 are classified as small, medium and large, respectively. As shown in the graph, the effect size of aesthetics on credibility $\left[(\mathrm{A} \rightarrow \mathrm{C})_{\mathrm{ES}}\right]$ is predominantly large, while that of usability on credibility $\left[(\mathrm{U} \rightarrow \mathrm{C})_{\mathrm{ES}}\right]$ is predominantly small. For example, with respect to WA | WB | $\mathrm{WC} \mid \mathrm{WD}$, the effect sizes $\left[(\mathrm{A} \rightarrow \mathrm{C})_{\mathrm{ES}}\right]$ for females are $(0.82 \mid$ $0.74|0.64| 0.61)$, respectively, while those for males are [0.59 | 0.56 | $0.40 \mid 0.14]$, respectively. On the other hand, the effect sizes $\left[(\mathrm{U} \rightarrow \mathrm{C})_{\mathrm{ES}}\right]$ for males are $[0.12|0.05| 0.002 \mid 0.04]$, respectively, while those for females are $(0.02|0.05| 0.02 \mid$ $0.05)$, respectively. Firstly, the predominantly all-high effect sizes $\left[(A \rightarrow C)_{E S}\right]$, except that with respect to mobile WD for the male group, compared to the predominantly all-small effect sizes $\left[(\mathrm{U} \rightarrow \mathrm{C})_{\mathrm{ES}}\right]$ for both groups, suggests that, irrespective of gender, aesthetics is more important than usability in predicting credibility. Secondly, with respect to $(\mathrm{A} \rightarrow \mathrm{C})_{\mathrm{ES}}$, the effect size is higher for females than for males.
This suggests that females care about aesthetics in evaluating credibility more than males do.

\section{H. Verification of Hypotheses}

In this subsection, we present the verification of our hypotheses.

1) The direct effect of Aesthetics on Credibility is stronger than the direct effect of Usability on Credibility: As shown in Fig. 3 and Fig. 4, the direct effects of aesthetics on credibility are relatively higher than the direct effects of usability on credibility at both the global and subgroup levels. For example, at the global level, the direct effects for $(A \rightarrow C)_{D I R}$ $\{0.71|0.64| 0.65 \mid 0.81\}$ with respect to $\{\mathrm{WA}|\mathrm{WB}| \mathrm{WC} \mid$ WD $\}$ are relatively higher than the direct effects for $(U \rightarrow C)_{D I R}$ $\{0.17|0.14| 0.02 \mid 0.22\}$. Similarly, for the male group, the direct effects for $(\mathrm{A} \rightarrow \mathrm{C})_{\mathrm{DIR}}[0.76|0.73| 0.74 \mid 0.86]$ are relatively higher than the direct effects for $(\mathrm{U} \rightarrow \mathrm{C})_{\text {DIR }}[0.27 \mid$ $0.20|-0.04| 0.27]$. Finally, for the female group, the direct effects for $(\mathrm{A} \rightarrow \mathrm{C})_{\text {DIR }}(0.64|0.55| 0.56 \mid 0.72)$ are relatively higher than the direct effects for $(\mathrm{U} \rightarrow \mathrm{C})_{\text {DIR }}(0.12|0.12| 0.11 \mid$ 0.18 ). Based on these results, we conclude that our first hypothesis (H1) is fully supported and replicated across both genders and all UI designs.

2) The direct effect of Aesthetics on Usability is stronger for the female group than for the male group: As shown in Fig. 4, contrary to our hypothesis, the influence of aesthetics on usability is higher for the male group [0.76 | $0.73|0.74|$ 0.86] than for the female group $(0.64|0.55| 0.56 \mid 0.72)$. However, the difference between each corresponding pair of path coefficients is not significant (see Table III). That being the case, our second hypothesis (H2) is not supported.

3) The direct effect of Aesthetics on Credibility is stronger for the female group than for the male group: As shown in Fig. 4, the direct effects of aesthetics on credibility for the female group $(0.72|0.70| 0.66 \mid 0.67)$ are not significantly higher than those for the male group [0.64 | $0.65|0.70| 0.49]$. Neither are the total effects shown in Fig. 5b and Fig. 5c for the female group $(0.80|0.76| 0.72 \mid 0.80)$ significantly higher than those for the male group [0.84 | $0.80|0.67| 0.73]$. Therefore, our third hypothesis (H3) is not supported.

4) The effect size of Aesthetics on Credibility is stronger for the female group than for the male group: As clearly shown in Fig. 7, for all four webpages, the effect sizes for the female group $(0.82|0.74| 0.64 \mid 0.61)$ are higher than effect sizes for the male group [0.59 | $0.56 \mid 0.40$ | 0.14$]$. Consequently, we conclude that our fourth hypothesis (H4) is fully supported and replicated across all UI designs.

5) The direct effect of Usability on Credibility is higher for the male group than for the female group: We presented the direct effects $\left[(U \rightarrow C)_{D I R}\right]$ for both genders in Fig. 4. As shown, for all four webpages, except for mobile WC, the 
direct effects for the male group $[0.27|0.20|-0.04 \mid 0.27]$ are stronger than those for the female group $(0.12|0.12| 0.11 \mid$ $0.18)$. However, it is only the difference between the first pair of path coefficients $(0.27, \mathrm{p}<0.001$ and $0.12, \mathrm{p}<0.05)$ for mobile WA that is significant at $p<0.05$. As a result of the non-replication of the finding across one or more UI designs, we conclude that our fifth hypothesis (H5), overall, is not fully supported. However, this calls for further investigation, especially given that three of the direct effects of usability on credibility are higher for males than for females (see Fig. 4).

6) The effect size of Usability on Credibility is higher for the male group than for the female group: Fig. 7 shows a plot of the effect sizes of usability on credibility. As shown, the effect sizes for the male group for all four webpages are [0.13 | $0.05|0.00| 0.04]$, while the effect sizes for the female group are $(0.02|0.05| 0.00 \mid 0.05)$. While the other corresponding pairs of effect sizes are virtually equal, the first effect size (for mobile WA) for the male group (0.13 - an almost medium effect size) is higher than that for the female group (0.02-a weak effect size). Based on this, we conclude that our sixth hypothesis (H6) is supported but not replicated. As such, overall, the sixth hypothessis (H6) is not fully supported.

7) The mediation of the direct effect of Aesthetics on Credibility by Usability is higher for the grid-layout mobile design than the list-layout mobile design: Fig. 6 shows a plot of the usability mediation of the effect of aesthetics on credibility. In the global, male and female models, the VAFs (a measure of mediation) for WA $|\mathrm{WB}| \mathrm{WC} \mid \mathrm{WD}$ are $\{0.14 \mid$ $0.11|0.00| 0.23\},[0.24|0.18| 0.00 \mid 0.32]$ and $(0.10|0.09|$ $0.00 \mid 0.16)$, respectively. As shown in the plot, at both the global and subgroup levels, the mediation by usability is higher for mobile WD than for mobile WA, WB and WC. In the global and male models, the usability mediations $(0.23$ and 0.32 , respectively) for mobile WD are partial. However, in the female model, though there is no mediation for mobile WD, as the VAF value (0.16) is less than 0.2, the VAF value for mobile WD (0.16) is higher than those for the other webpages $(0.10,0.09$ and 0.00$)$. Consequenetly, given that the usability mediation of the effect of aesthetics on credibility is higher for mobile WD (a grid-layout design) than for the other webpages (list-layout designs), we conclude that our seventh hypothesis (H7) is supported and replicated across both genders. However, given that for the female model, the VAF value is less than 0.2 , there is a need for further investigation of $\mathrm{H} 7$.

8) The mediation of the direct effect of Aesthetics on Credibility by Usability is stronger for the male group than for the female group. As shown in Fig. 6, the VAF values for mobile WA | WB | WC | WD are higher for the male group $[0.24|0.18| 0.00 \mid 0.32]$ than for the female group $(0.10 \mid$ $0.09|0.00| 0.16)$, except for mobile WC where there is no mediation. Therefore, our eighth hypothesis (H8) is supported and replicated across (three) UI designs.

\section{DISCUSSION}

We have presented the results of our study on the interplay of aesthetics, usability and credibility in the judgment of mobile web designs. For quick and easy reference, we have summarized in Table IV all of our main findings, including an additional finding $\left(\mathrm{H}^{\mathrm{a}}\right)$, which contradicts our second hypothesis (H2). First, four of our hypotheses (H1, H4, H7 and H8) are supported and replicated across all four UI designs. Second, two of our hypotheses (H5 and H6) are supported with respect to one UI design only, but are not replicated across the other three UI designs. Third, two of the hypotheses (H2 and H3) are neither supported nor replicated. Finally, the opposite of our second hypothesis (i.e., H9a) is supported and replicated across all four UI designs.

\section{A. H1: Validation of Hypotheses}

In this subsection, we discuss the validation and nonvalidation of the hypotheses summarized in Table IV.

TABLE IV. SUMMARY OF VALIDATED HyPOTHESES

\begin{tabular}{|c|c|c|}
\hline No. & Hypothesis & Remark \\
\hline H1 & $\begin{array}{l}\text { The direct effect of Aesthetics on Credibility is } \\
\text { stronger than the direct effect of Usability on } \\
\text { Credibility. }\end{array}$ & $\begin{array}{l}\text { Supported } \\
\text { and } \\
\text { replicated }\end{array}$ \\
\hline H2 & $\begin{array}{l}\text { The direct effect of Aesthetics on Usability is } \\
\text { stronger for the female group than for the male } \\
\text { group. }\end{array}$ & $\begin{array}{c}\text { Not } \\
\text { Supported }\end{array}$ \\
\hline H3 & $\begin{array}{l}\text { The direct effect of Aesthetics on Credibility is } \\
\text { stronger for the female group than for the male } \\
\text { group. }\end{array}$ & $\begin{array}{l}\text { Not } \\
\text { Supported }\end{array}$ \\
\hline H4 & $\begin{array}{l}\text { The effect size of Aesthetics on Credibility is } \\
\text { higher for the female group than for the male } \\
\text { group. }\end{array}$ & $\begin{array}{l}\text { Supported } \\
\text { and } \\
\text { replicated }\end{array}$ \\
\hline H5 & $\begin{array}{l}\text { The direct effect of Usability on Credibility is } \\
\text { stronger for the male group than for the female } \\
\text { group. }\end{array}$ & $\begin{array}{l}\text { Supported } \\
\text { but not } \\
\text { replicated }\end{array}$ \\
\hline H6 & $\begin{array}{l}\text { The effect size of Usability on Credibility is higher } \\
\text { for the male group than for the female group. }\end{array}$ & $\begin{array}{l}\text { Supported } \\
\text { but not } \\
\text { replicated }\end{array}$ \\
\hline H7 & $\begin{array}{l}\text { The mediation of the direct effect of Aesthetics on } \\
\text { Credibility by Usability is higher for the grid- } \\
\text { layout mobile design than the list-layout mobile } \\
\text { designs. }\end{array}$ & $\begin{array}{l}\text { Supported } \\
\text { and } \\
\text { replicated }\end{array}$ \\
\hline H8 & $\begin{array}{l}\text { The mediation of the direct effect of Aesthetics on } \\
\text { Credibility by Usability is stronger for the male } \\
\text { group than for the female group. }\end{array}$ & $\begin{array}{l}\text { Supported } \\
\text { and } \\
\text { replicated }\end{array}$ \\
\hline $\mathrm{H9}^{\mathbf{a}}$ & $\begin{array}{l}\text { The direct effect of Aesthetics on Usability is } \\
\text { stronger for the male group than for the female } \\
\text { group. }\end{array}$ & $\begin{array}{l}\text { Supported } \\
\text { and } \\
\text { Replicated }\end{array}$ \\
\hline
\end{tabular}

Note: $\mathrm{H} 9^{\mathrm{a}}$ (opposite of $\mathrm{H} 2$ ) was not pre-formulated but turned out to be true.

1) H1: The direct effect of Aesthetics on Credibility is stronger than the direct effect of Usability on Credibility: As presented in Table IV, we showed through path modeling that our first hypothesis (H1) is supported by replicating it across gender and all four UI designs. This finding replicates our prior finding in our study which is based on two different cultures [7]. Thus, with the validation of this hypothesis in this 
paper, we have provided more empirical evidence in the mobile web domain (which cuts across gender) by confirming that perceived aesthetics is a stronger determinant than perceived usability in predicting the perceived credibility of a mobile website. Moreover, this finding replicates prior findings in the web domain. For example, Fogg et al. [9] found that design look was the most important factor in evaluating web credibility. In their qualitative study among 2,684 participants, they found that design look was mentioned in $46.1 \%$ of participants' comments. However, it is noteworthy that while we have found aesthetics to be a stronger predictor of mobile web credibility, as compared to usability, our finding does not suggest that usability is not important (and should not be considered) when designing mobile websites. Off course, usability is important in the user experience, especially in the mobile domain where the relatively small screen size can pose a serious threat to the effective and efficient use of mobile applications [17]. As we have shown in the global and subgroup models (in Fig. 3 and Fig. 4), usability plays a role in the judgment of credibility as well, especially if a user has to actually use a website for some time to accomplish a task. In three of the presented mobile designs (WA, WB and WD), we have also presented empirical evidence, which shows that usability (though not as strongly as aesthetics) influences the credibility of a mobile website. According to Fogg et al. [83], usability (i.e., making sites easy to use) will equally enhance the credibility of a website. In summary, our finding suggests that, while ensuring that mobile web applications are designed to be easily usable given our awareness of the relatively small size of the mobile screen and the usability challenges it poses in human-computer interaction, designers should also endeavor to cater to users' hedonic needs by designing their websites to be visually appealing. This has the potential of attracting more users to their websites at the visceral level of judgment, which is based on first impression [1], [84].

2) H2: The direct effect of Aesthetics on Usability is stronger for the female group than for the male group: Our second hypothesis $(\mathrm{H} 2)$, as presented in Table IV, is not supported. The influence of aesthetics on usability is aptly regarded as the "aesthetic-usability effect" [31], a cognitive bias in human-computer interaction, is also regarded to as the "halo effect" [57]. Contrary to our hypothesis, in our study the halo effect is stronger for the male group than the female group, as stated in $\mathrm{H}^{\mathrm{a}}$. This is reflected in the coefficient of determination of both subgroup models (see Fig. 4), with aesthetics in the male model $\left(\mathrm{R}^{2}=[0.58|0.53| 0.34 \mid 0.74]\right)$ explaining the variance in usability more than in the female model $\left(\mathrm{R}^{2}=(0.40|0.30| 0.32 \mid 0.52)\right)$. This suggests that: (1) females' usability perception is more difficult to predict using aesthetics perception compared to males'; and (2) there are other factors (which are not captured in our model) that may account for the variance in perceived usability, especially for the female group. This implies that there is a need for more research to identify and understand these other factors than aesthetics (e.g., information design, advertising, etc. [9]), which may impact usability in the mobile domain. Furthermore, the unexpected finding that perceived aesthetics influences perceived usability more strongly for the male group than for the female group suggests that the aestheticusability halo effect is stronger in males than in females.

3) H3:The direct effect of Aesthetics on Credibility is stronger for the female group than for the male group: Based on the finding that females are more likely to be motivated by intrinsic factors in their judgment than males [36], we had hypothesized that the influence of aesthetics on credibility will be stronger for the female group than for the male group (H3). However, our multigroup analysis showed no support for this hypothesis. However, there might be a need for further future research to find out whether other factors than gender (such as culture, age, etc.) may moderate the relationship between perceived aesthetics and perceived credibility.

4) H4: The effect size of Aesthetics on Credibility is stronger for the female group than for the male group: The result of our effect-size analysis (see Fig. 7) reveals that the effect size of aesthetics on credibility is stronger for the female group than for the male group (H4). It is interesting to note that while our third hypothesis (H3), which concerns the direct effect of aesthetics on credibility, is not supported, our fourth hypothesis (H4), which concerns the effect size of aesthetics on credibility, is supported for all four UI designs. This suggests that, in an actual setting, perceived aesthetics may have a stronger influence on the perceived credibility of websites for the female group than it does for the male group. This finding replicates the notion that females are more concerned about intrinsic factors than males in judging products, such as websites [36].

5) H5: The direct effect of Usability on Credibility is higher for the male group than for the female group: The fifth hypothesis (H5) is only supported with respect to one of the UI designs. Specifically, we found a significant difference between males and females with respect to mobile WA only, with usability having a higher effect on credibility for males than for females (see Fig. 4 and Table III). However, given that this finding is not replicated among any of the other web designs than mobile WA, we conclude that, overall, our hypothesis (H5) is not supported. However, given that there is evidence that supports the fifth hypothesis (H5), with respect to mobile WA, there might be a need for further investigation to be carried out to verify this hypothesis, especially given that all of the three significant usability-credibility relationships are higher for the male group than for the female group.

6) H6: The effect size of Usability on Credibility is higher for the male group than for the female group: The sixth hypothesis on the effect size of usability on credibility is only 
supported with respect to mobile WA; it is not replicated across the other three UI designs. Specifically, we found that the effect size with respect to mobile WA is larger for the male group (0.12 - almost medium ES) than for the female group (0.02-small ES). This is also evident in the significantly different direct effects when the male group $(\beta=$ $0.27, p<0.001)$ is compared to the female group $(\beta=0.12, p$ $<0.05$ ), as indicated by bold fonts in Fig. 4. In practical terms, this suggests that, in the judgment of credibility with respect to mobile design, in general (or, in particular, low-aesthetic mobile web design), usability may matter more to males than it does to females. However, the non-replication of this finding (significant difference between males and females with respect to the $(\mathrm{U} \rightarrow \mathrm{C})_{\text {DIR }}$ relationship) across two or more UI designs makes it difficult for us to conclude that our sixth hypothesis (H6) is supported. As a result, we conclude that, overall, our sixth hypothesis (H6) is not supported. However, given the evidence in favor of this hypothesis with respect to mobile WA, further investigations may need to be carried out in future research to valiadte or invalidate it.

7) H7: The mediation of the direct effect of Aesthetics on Credibility by Usability is higher for the grid-layout mobile design than the list-layout mobile design: Our seventh hypothesis states that, with respect to organization of information or content in mobile web design, usability will mediate the effect of aesthetics on credibility more for a grid layout (mobile WD) than for the list layouts (mobile WA, WC and WC). The reasoning behind this hypothesis is that, in a prior qualitative study, Oyibo, Ali and Vassileva [60] found that the grid-layout design is perceived as more usable than the list-layout design by both genders due to how well-spaced out the icon-based items were on the screen compared to the list-based items, which are relatively closer and almost touching. Specifically, regarding mobile WD, a participant in their study commented: "The buttons would probably be easy to press with my stubby fingers without accidentally hitting the wrong one" (p. 82) [60]. As expected (see Fig. 6), we found that, for both genders, the mediation of usability of the effect of aesthetics on credibility is higher for mobile WD than for the other webpages. Thus, our seventh hypothesis (H7) is supported and replicated across gender. This finding suggests that the more easy to use (e.g., navigate) a mobile website is perceived, the higher the possibility of usability mediating the effect of perceived aesthetics on perceived credibility.

8) H8: The mediation of the direct effect of Aesthetics on Credibility by Usability is stronger for the male group than for the female group. As shown in Fig. 6, the usability mediation of the effect of aesthetics on credibility is higher for males than for females with respect to all the webpages, except for mobile WC. Specifically, for mobile WA and WD, there is partial usability mediation for the male group but none for the female group. Consequently, our eighth hypothesis (H8) is supported and replicated. This suggests that males may be more likely concerned about usability (an instrumental attribute) than females in the judgment of credibility, as found in prior research [36], [60]. However, this may need further investigation, for example, given that the hypothesis is not verified with respect to all four UIs. Aside, readers might be interested in knowing the likely reason for the lack of usability mediation of the effect of aesthetics on credibility with respect to mobile WC as compared to the other mobile webpages (WA, WB and WD). As a result, we provide a plausible explanaation. In the global model (Fig. 5a), we see that in the $(\mathrm{A} \rightarrow \mathrm{C})_{\mathrm{IND}}$ effects for mobile $\{\mathrm{WA}|\mathrm{WB}| \mathrm{WC} \mid \mathrm{WD}\}$, i.e, $\{0.12|0.09| 0.01 \mid 0.18\}$, respectively, the indirect effect for mobile WC is virtually zero (non-significant). Similarly, in the male model (Fig. 5b), we see that in the $(A \rightarrow C)_{\text {IND }}$ effects [0.21 $0.15|-0.03| 0.23]$ for all four UIs, the indirect effect for mobile WC is virtually zero (non-significant). Finally, in the female model (Fig. 5c), we see that in the $(A \rightarrow C)_{\text {IND }}(0.08$ | $0.07|0.06| 0.13$ ) for all four UIs, the indirect effect for mobile WC is least and non-significant as well. One possible explanation for this finding (the non-significance of $(A \rightarrow C)_{\text {IND }}$ for mobile WC) is that mobile WC was presented to all of the participants first in the online survey. It appears that, upon their initial encounter with this version of the hypothetical website (WC), the only criterion participants used in judging credibility was aesthetics; usability, as our path models shows was not used (i.e., perceived usability neither affected perceived credibility nor mediated the direct effect $\left[(\mathrm{A} \rightarrow \mathrm{C})_{\mathrm{DIR}}\right]$, with respect to $\mathrm{WC}$, as evident in the nonsignificant direct effect $\left[(\mathrm{U} \rightarrow \mathrm{C})_{\mathrm{DIR}}\right]$ shown in Fig. 3 and Fig. 4). In other words, in the context of the elaboration likelihood model [85], both males and females used, completely, the peripheral route, i.e., the short-circuited path $\left[(\mathrm{A} \rightarrow \mathrm{C})_{\mathrm{DIR}}\right]$, to judge credibility. This is consistent with the prior finding that the judgment of credibility of websites is made within the first few seconds of users' coming in contact with the user interface and it is mainly informed by visual appeal. Lindgaard et al. [84] found that when a person opens a website, the first impression, predicated on the visual appeal of the site, is made within the first 50 milliseconds. According to Robins and Holmes [1], this first impresiion determines whether a person will continue using (e.g., browsing) a website or abandon it for another website. Consequently, in our study, it is very likely that the first impression, based on visual appeal, must have shifted participants' attention away from usability-related attributes and concerns. Thus, with respect to mobile WC, presented first in the online survey, we find that perceived usability neither influences nor mediates the influence of perceived aesthetics on perceived credibility - a finding which cuts across gender.

\section{B. Implications and Contributions}

In summary, we would like to briefly discuss the overarching implication of our findings in the context of our research questions and summarize our contributions. Overall, the results of our PLSPM analysis show that, irrespective of the level of aesthetic treatment of mobile websites, the layout of 
the UI design and gender, aesthetics is a stronger determinant of credibility than usability is. This implies that, in the mobile web domain, the hedonic need of users has to be catered to as well by designers [86], as designing websites that are only usable, but lack visual appeal, may not be able to attract a critical mass of users as a result of poor first impressions made by the websites on visitors. Therefore, mobile web designers should ensure their sites are visually appealing, as perceived aesthetics, at the visceral level of website evaluation, is stronger than perceived usability in predicting web credibility, as found in prior research [1], [7], [9], [87] on web design.

In conclusion, our main contributions to the body of knowledge can be summarized as follows:

1. We showed that, in the evaluation of mobile websites, perceive aesthetics and perceived usability combined can account for up to and over $50 \%$ of the variance in perceived credibility.

2. We showed that, regardless of gender and layout of UI design, perceived aesthetics is stronger than perceived usability in predicting the perceived credibility of a mobile website.

3. We presented new empirical evidence regarding the moderating effect of gender and design layout in the interrelationships among the three web design constructs of interest. They include the following:

a. The aesthetic-usability effect is stronger for males than for females.

b. The effect size of aesthetics on credibility is larger for females than for males.

c. The usability mediation of the effect of aesthetics on credibility is larger for males than for females.

d. The usability mediation of the effect of aesthetics on credibility is larger for a gridlayout than for a list-layout mobile website.

Finally, our study, to the best of our knowledge, is the first of its kind in the mobile web domain that has presented empirical evidence on the interrelationships among the three important web design constructs (aesthetics, usability and credibility) in a replicated fashion across different UI designs and gender, using a multicultural population sample that cuts across five continents. This opens up the possibility of a broader generalization of findings.

\section{LIMITAION AND FUTURE WORK}

The main limitation of our study is that the measurement of the three web design constructs (perceived aesthetics, perceived usability and perceived credibility), which we investigated, was based on perception and not actual use of the mobile websites. This might have impacted our findings, thereby limiting the scope of our findings to the visceral level of perception, as our current findings may differ in the context of actual use of the mobile websites. A second limitation of our study is that, for convenience in answering the online survey questions, most participants might have viewed the mobile web designs on a desktop rather than a mobile device. This might have affected their perception of the mobile web designs and the way they responded. Finally, the fixed order of presentation of the mobile web designs in the online survey must have also impacted our findings to a certain degree, as we pointed out when discussing the eighth hypothesis. However, in future work, we look forward to addressing some of these limitations, including investigating the qualitative response of users to the four web designs covered in this paper and how it may differ across culture, age, gender and UI design.

\section{CONCLUSION}

In this paper, we presented a partial least square path model of the interrelationships among three key web design constructs of mobile websites. Specifically, we attempt to uncover which of the key determinants of web credibility (perceived aesthetics or perceived usability) has a stronger influence on perceived credibility in the mobile domain, and the role UI design and gender play. Using a multicultural sample of 526 participants from five continents (Africa, Asia, North America, South America and Europe) and four systematically designed mobile websites, our path model reveals that, irrespective of UI design and gender, perceived aesthetics has a stronger effect than perceived usability on the perceived credibility of a mobile website. Secondly, our model shows that the effect size of perceived aesthetics on perceived credibility is larger for females than for males. Thirdly, our model shows that the aesthetic-usability effect and the usability mediation of the effect of perceived aesthetics on perceived credibility are stronger for males than for females. Finally, our model shows that the usability mediation of the direct effect between aesthetics and credibility may depend on the layout of content on the screen of the mobile device. Specifically, there is a stronger usability mediation of the effect of aesthetics on credibility for the grid-layout mobile design than the list-layout mobile designs. This suggests that users may be more cognizant of usability concerns in the judgment of credibility in a grid-layout mobile design than in a list-layout mobile design. However, this finding requires further investigation.

The overall implication of our findings is that, while usability (a utilitarian attribute) is of great concern in the mobile domain given the relatively small size of the mobile device, designers should not lose sight of the need to satisfy the hedonic needs of users as well, as users, as we have shown, perceive aesthetics (visual appeal) as very important in the whole user experience and in the overall judgment of web credibility, especially at the visceral (non-cognitive) level of perception. In conclusion, our findings suggest that, although, in some specific use cases, one of the two web design attributes may be given priority over the other, in general, there is a need for designers to strike a balance between hedonic and utilitarian concerns [88], while specifically paying close attention to visual aesthetics - which the user first comes in contact with in human-computer interaction — and gender differences as well.

\section{ACKNOWLEDGEMENT}

The authors would like to thank the Natural Sciences and Engineering Research Council of Canada (NSERC) for providing the funding which made this research possible. 


\section{REFERENCES}

[1] D. Robins and J. Holmes, "Aesthetics and credibility in web site design," Information Processing \& Management, vol. 44, no. 1, pp. 386-399, 2008.

[2] B. Fogg, "What Makes a Website Credible?," Stanford University, $2003 . \quad$ [Online]. Available: http://static.lukew.com/web_credibility_lecture.pdf. [Accessed: 17Feb-2017].

[3] Trust Verified, "The Importance of Website Credibility," Trust Verified. [Online]. Available: http://trust-verified.org/theimportance-of-website-credibility/. [Accessed: 17-Feb-2017].

[4] B. J. Fogg, J. Marshall, O. Laraki, A. Osipovich, C. Varma, N. Fang, J. Paul, A. Rangnekar, J. Shon, P. Swani, M. Treinen, and C. Hall, "What Makes Web Sites Credible? A Report on a Large Quantitative Study CHI 2001," in Sigchi'01, 2001, pp. 61-68.

[5] T. Lavie and N. Tractinsky, "Assessing dimensions of perceived visual aesthetics of web sites," International Journal of HumanComputer Studies, vol. 60, no. 3, pp. 269-298, Mar. 2004.

[6] C.-H. Liu, C.-F. Lee, and H.-L. Lee, "The Influence of HCI Design Aesthetics on Website Creditability - Using on Online Banking Website Interfaces as an Example," in Design Computing and Cognition, 2010, pp. 1-20.

[7] K. Oyibo and J. Vassileva, "The Interplay of Aesthetics, Usability and Credibility in Mobile Websites and the Moderation by Culture," in Brazilian Symposium on Human Factors in Computer Syatems, 2016

[8] A. Sonderegger, A. Uebelbacher, M. Pugliese, and J. Sauer, "The Influence of Aesthetics in Usability Testing: The Case of Dualdomain Products," Proceedings of the SIGCHI Conference on Human Factors in Computing Systems, pp. 21-30, 2014.

[9] B. J. Fogg, C. Soohoo, D. R. Danielson, L. Marable, J. Stanford, and E. R. Tauber, "How do users evaluate the credibility of Web sites?: a study with over 2,500 participants," in Proceedings of the 2003 conference on Designing for user experiences, 2003, pp. 1-15.

[10] D. Cyr, "Gender and website design across cultures," 17th European Conference on Information Systems, pp. 279-291, 2009.

[11] N. Tractinsky, "Aesthetics and apparent usability: Empirically assessing cultural and methodological issues.," Conference on Human Factors in Computing Systems, pp. 115-122, 1997.

[12] Z. Gócza, "Myth \# 25: Aesthetics are not important if you have good usability." [Online]. Available: http://uxmyths.com/post/1161244116/myth-25-aesthetics-are-notimportant-if-you-have-good-us. [Accessed: 02-Apr-2016].

[13] N. Tractinsky, A. S. Katz, and D. Ikar, "What is beautiful is usable," Interacting with computers, vol. 13, no. 2, pp. 127-145, 2000.

[14] M. Hassenzahl and A. Monk, "The Inference of Perceived Usability From Beauty," Human-Computer Interaction, vol. 24, no. March 2015, pp. 37-41, 2010.

[15] A. David and P. Glore, "The Impact of Design and Aesthetics on Usability, Credibility, and Learning in Online Courses," Proceedings of World Conference on E-Learning in Corporate, Government, Healthcare, and Higher Education 2010, no. 2004, p. 42, 2010.

[16] N. E. Youngblood and J. MacKiewicz, "A usability analysis of municipal government website home pages in Alabama," Government Information Quarterly, vol. 29, no. 4, pp. 582-588, 2012

[17] M. Chae and J. Kim, "Do size and structure matter to mobile users? An empirical study of the effects of screen size, information structure, and task complexity on user activities with standard web phones," Behaviour \& Information Technology, vol. 23, no. 3, pp. 165-181, 2004.

[18] N. Kumar, "Usability for the new PC: mobile devices," Third Annual Symposium Theory and Research in HCI, 2012.

[19] Interaction Design Foundation, "The 7 Factors that Influence User Experience." [Online]. Available: https:/www.interactiondesign.org/literature/article/the-7-factors-that-influence-userexperience?utm_content=buffer4f582\&utm_medium=social\&utm_s ource=facebook.com\&utm_campaign=buffer. [Accessed: 27-Dec2016].

[20] S. Mahlke and M. Thüring, "Studying antecedents of emotional experiences in interactive contexts," Proceedings of the SIGCHI conference on Human factors in computing systems - CHI '07, pp.
915-918, 2007.

[21] T. Lavie and N. Tractinsky, "Assessing dimensions of perceived visual aesthetics of web sites," International Journal of Human Computer Studies, vol. 60, no. 3, pp. 269-298, 2004.

[22] N. Tractinsky, "Aesthetics and apparent usability: empirically assessing cultural and methodological issues," in Proceedings of the ACM SIGCHI Conference on Human factors in computing systems, 1997, pp. 115-122.

[23] K. Oyibo and J. Vassileva, "What Drives Perceived Usability in Mobile Web Design : Classical or Expressive Aesthetics?," in 19th International Conference on Human-Computer Interaction, 2017, pp. 445-462.

[24] D. A. Norman, The psychology of everyday things. Basic Books, 1988.

[25] M. Kurosu and K. Kashimura, "Apparent Usability vs. Inherent Usability: Experimental Analysis on the Determinants of the Apparent Usability," in Conference Companion on Human Factors in Computing Systems, 1995, pp. 292-293.

[26] O. Taebi, H. Aldabbas, and M. Clarkson, "Designing for Usability or Aesthetics in E-commerce Websites," 2012.

[27] J. Nielsen, Usability Engineering, vol. 25, no. 3. Elsevier, 1993.

[28] M. Kurosu, K. Kashimura, and O. F. Creativity, "Apparent usability vs. inherent usability: experimental analysis on the determinants of the apparent usability," Proceedings of the ACM Conference on Human Factors in Computing Systems, pp. 292-293, 1995.

[29] K. Reinecke, T. Yeh, L. Miratrix, R. Mardiko, Y. Zhao, J. Liu, and K. Z. Gajos, 'Predicting users' first impressions of website aesthetics with a quantification of perceived visual complexity and colorfulness," Proceedings of the SIGCHI Conference on Human Factors in Computing Systems - CHI '13, pp. 2049-2058, 2013.

[30] K. E. Kripintiris, "Web aesthetics and usability: An empirical evaluation of white space," ProQuest Dissertations and Theses, $\mathrm{p}$. 65, 2008.

[31] W. Lidwell, K. Holden, and J. Butler, "Universal Principles of Design," Universal principles of design: 125 ways to enhance usability, influence perception, increase appeal, make beter design decisions, and teach through design., pp. 1-271, 2010.

[32] D. Cyr and C. Bonanni, "Gender and website design in e-business," International Journal of Electronic Business, vol. 3, no. 6, p. 565, 2005.

[33] S. Ferebee, "The influence of gender and involvement level on the perceived credibility of web sites," Lecture Notes in Computer Science (including subseries Lecture Notes in Artificial Intelligence and Lecture Notes in Bioinformatics), vol. 5033 LNCS, pp. 279$282,2008$.

[34] S. J. Simon, "The impact of culture and gender on web sites: an empirical study," SIGMIS Database, vol. 32, no. 1, pp. 18-37, 2001.

[35] J. Meyers-Levy, "Gender Differences in Information Processing: A Selectivity Interpretation," Cognitive and Affective Responses to Advertising, pp. 219-260, 1989.

[36] Y. Sun, K. H. Lim, C. Jiang, J. Z. Peng, and X. Chen, "Do males and females think in the same way? An empirical investigation on the gender differences in Web advertising evaluation," Computers in Human Behavior, vol. 26, no. 6, pp. 1614-1624, 2010.

[37] K. Oyibo, Y. S. Ali, and J. Vassileva, "Gender Difference in the Credibility Perception of Mobile Websites: A Mixed Method Approach," in Proceedings of the 2016 Conference on User Modeling Adaptation and Personalization - UMAP '16, 2016, pp. 75-84.

[38] A. Harley, "Trustworthiness in Web Design: 4 Credibility Factors," Nielsen Norman Group (NN/g), 2016. [Online]. Available: https://www.nngroup.com/articles/trustworthy-design.

[39] D. Cyr and H. Trevor-Smith, "Localization of web design: An empirical comparison of German, Japanese, and United States web site characteristics," Journal of the American Society for Information Science and Technology, vol. 55, no. 13, pp. 11991206,2004

[40] B. J. Fogg, J. Marshall, O. Laraki, A. Osipovich, C. Varma, N. Fang, J. Paul, A. Rangnekar, J. Shon, P. Swani, M. Treinen, and C. Hall, "What Makes Web Sites Credible? A Report on a Large Quantitative Study CHI 2001,” pp. 61-68, 2001.

[41] D. S. Soper, "User Interface Design and the Halo Effect: Some Preliminary Evidence," in Twentieth Americas Conference on Information Systems, Savannah, 2014, pp. 1-11. 
[42] K. Reinecke and A. Bernstein, "Improving performance, perceived usability, and aesthetics with culturally adaptive user interfaces," ACM Transactions on Computer-Human Interaction, vol. 18, no. 2 , pp. 1-29, 2011.

[43] B. J. Fogg, Persuasive Technology: Using Computers to Change What We Think and Do. Morgan Kaufmann, 2003.

[44] B. J. Fogg, "Prominence-interpretation theory: Explaining how people assess credibility online," Conference on Human Factors in Computing Systems - Proceedings, pp. 722-723, 2003.

[45] C. Sartwell, "Beauty," in The Stanford Encyclopedia of Philosophy, Winter 201., E. N. Zalta, Ed. Metaphysics Research Lab, Stanford University, 2016.

[46] E. Burns Coleman, C. Hartney, and Z. Alderton, "Defining 'Social Aesthetics," Aesthetics, vol. 23, no. 1, pp. 1-11, 2013.

[47] H. Caygill, "Aesthetics and civil society: theories of art and society 1640 - 1790," University of Sussex, 1982.

[48] L. Mastin, "The Basics of Philosophy," 2008. [Online]. Available: http://www.philosophybasics.com/branch_aesthetics.html. [Accessed: 23-Sep-2017].

[49] D. Burnham, "Immanuel Kant: Aesthetics," Internet Encyclopedia of Philosophy. [Online]. Available: http://www.iep.utm.edu/kantaest/. [Accessed: 12-Nov-2017].

[50] Kenaan, "Philosophy's Moods: The Affective Grounds of Thinking," Igarss 2014, no. 1, pp. 1-5, 2014.

[51] N. Zangwill, "Aesthetic Judgment," in The Stanford Encyclopedia of Philosophy, Fall 2014., E. N. Zalta, Ed. Metaphysics Research Lab, Stanford University, 2014.

[52] N. Tractinsky, "Visual Aesthetics," Interaction-Design, pp. 1-71, 2002.

[53] A. Sonderegger, J. Sauer, and J. Eichenberger, "Expressive and classical aesthetics: two distinct concepts with highly similar effect patterns in user-artefact interaction," Behaviour \& Information Technology, vol. 33, no. 11, pp. 1180-1191, 2014.

[54] N. Tractinsky, "Toward the study of aesthetics in information technology," ICIS 2004 Proceedings, p. 62, 2004.

[55] V. Venkatesh and F. D. Davis, "A theoretical extension of the technology acceptance model: Four longitudinal field studies," Information Systems Research, vol. 46, no. 2, pp. 186-204, 2000.

[56] K. A. Butler, "Usability Engineering Turns 10," Interactions, vol. 3, no. 1 , pp. 58-75, 1996.

[57] A. Sonderegger and J. Sauer, "The influence of design aesthetics in usability testing: Effects on user performance and perceived usability," Applied Ergonomics, vol. 41, no. 3, pp. 403-410, 2010.

[58] M. Hassenzahl, "The interplay of beauty, goodness, and usability in interactive products," Human-Computer Interaction, vol. 19, no. 4, pp. 319-349, 2004.

[59] Y. M. Li and Y. S. Yeh, "Increasing trust in mobile commerce through design aesthetics," Computers in Human Behavior, vol. 26, no. 4, pp. 673-684, 2010.

[60] K. Oyibo, Y. S. Ali, and J. Vassileva, "Gender Difference in the Credibility Perception of Mobile Websites: A Mixed Method Approach," in User Modeling, Adaptation and Personalization (UMAP 2016), 2016, pp. 1-10.

[61] Y.-M. Li and Y.-S. Yeh, "Increasing trust in mobile commerce through design aesthetics," Computers in Human Behavior, vol. 26, no. 4, pp. 673-684, 2010.

[62] K. Oyibo and J. Vassileva, "The Interplay of Aesthetics, Usability and Credibility in Mobile Websites and the Moderation by Culture," 2016.

[63] Y. Neumark, L. Flum, C. Lopez-Quintero, and R. Shtarkshall, "Quality of online health information about oral contraceptives from Hebrew-language websites," Israel Journal of Health Policy Research, vol. 1, no. 1, pp. 1-10, 2012.

[64] M. Miche and M. Noirhomme-fraiture, "Gender differences in graphic design for the Web Categories and Subject Descriptors," Revista Eletrônica de Sistemas de Informação, vol. 5, no. 1, 2009.

[65] G. Moss and R. Gunn, "Gender differences in website design: Implications for education," Int Conf on Cybernetics and Information Technologies, Systems and Applications/Int Conf on Computing, Communications and Control Technologies, Vol 1, pp. 54-59, 2007.

[66] S. Djamasbi, T. Tullis, J. Hsu, E. Mazuera, K. Osberg, and J. Bosch, "Gender preferences in web design: usability testing through eye tracking," Proceedings of the Americas Conference on Information Systems, pp. 1-8, 2007.

[67] K. Oyibo, Y. S. Ali, and J. Vassileva, "An empirical analysis of the perception of mobile website interfaces and the influence of culture," in Proceedings of the Personalization in Persuasive Technology Workshop, Persuasive Technology 2016, 2016, vol. 1582, pp. 44-56.

[68] T. Ramakrishnan, V. Prybutok, and D. A. Peak, "The moderating effect of gender on academic website impression," Computers in Human Behavior, vol. 35, pp. 315-319, 2014

[69] L. Xue and C. C. Yen, "Towards female preferences in design - A pilot study," International Journal of Design, vol. 1, no. 3, pp. 1127, 2007.

[70] M. Arcand and J. Nantel, "What drives ease of use perceptions and behavioral intentions towards websites for men and women? A study of French-Canadian Internet Users," Management and Avenir, vol. 2, no. 32, pp. 154-172, 2010.

[71] E. Shinners, "Effects of The \&quot; What is Beautiful is Good \&quot; Stereotype on Perceived Trustworthiness," Shinners $U W-L$ Journal of Undergraduate Research XII, pp. 1-5, 2009.

[72] K. Oyibo, Y. S. Ali, and J. Vassileva, "An empirical analysis of the perception of mobile website interfaces and the influence of culture," in CEUR Workshop Proceedings, 2016, vol. 1582, pp. 4456.

[73] P. van Schaik and J. Ling, "The role of context in perceptions of the aesthetics of web pages over time," International Journal of Human-Computer Studies, vol. 67, no. 1, pp. 79-89, 2009.

[74] A. De Angeli, A. Sutcliffe, and J. Hartmann, "Interaction, usability and aesthetics: What influences users' preferences?," in Proceedings of the 6th Conference on Designing Interactive Systems, 2006, no. November, pp. 271-280.

[75] S. P. S. Setterstrom, "Assessing credibility and aesthetic perception across different exposure times on a health care information website."

[76] L. Bergkvist and J. R. Rossiter, "The Predictive Validity of Multiple-Item Versus Single-Item Measures of the Same Constructs," Journal of Marketing Research, vol. 44, no. 2, pp. 175-184, 2007.

[77] L. Bergkvist, "Appropriate use of single-item measures is here to stay," Marketing Letters, vol. 26, no. 3, pp. 245-255, 2015.

[78] G. Sanchez, "PLS Path Modeling with R," Berkley: Trowchez Editions, 2013.

[79] J. F. Hair, G. T. M. Hult, C. M. Ringle, and M. Sarstedt, A Primer on Partial Least Squares Structural Equation Modeling (PLS-SEM). Sage Publications, Inc, Washington DC, 2014.

[80] K. J. Preacher and K. Kelley, "Effect size measures for mediation models: quantitative strategies for communicating indirect effects," Psychological Methods, vol. 16, no. 2, pp. 93-115, 2011.

[81] J. Cohen, "Statistical power analysis for the behavioral sciences," Statistical power analysis for the behavioral sciences (rev. ed.)., vol. 2nd, 1988.

[82] J. A. Durlak, "How to select, calculate, and interpret effect sizes," Journal of Pediatric Psychology, vol. 34, no. 9, pp. 917-928, 2009.

[83] J. A. Jacko and A. Sears, "The human-computer interaction handbook: fundamentals, evolving technologies, and emerging applications," The human-computer interaction handbook, p. 1277, 2003.

[84] G. Lindgaard, G. Fernandes, C. Dudek, and J. Brown, "Attention web designers: You have 50 milliseconds to make a good first impression!," Behaviour \& Information Technology, vol. 25, no. 2, pp. 115-126, 2006.

[85] R. E. Petty, J. T. T. Cacioppo, and R. E. Nisbett, "The elaboration likelihood model of persuasion," Advances in experimental social psychology, vol. 19, no. 1, pp. 123-205, 1986.

[86] M. Hassenzahl, "The Thing and I: Understanding the Relationship Between User and Product," Funology, vol. 3, pp. 31-42, 2003.

[87] K. Oyibo, R. Orji, and J. Vassileva, "The Influence of Personality on Mobile Web Credibility," in Adjunct Proceedings of User Modeling, Adaptation and Personalization (UMAP 2017), 2017.

[88] U. K. Yusof, L. K. Khaw, H. Y. Ch'ng, and B. J. Neow, "Balancing between usability and aesthetics of Web design," Proceedings 2010 International Symposium on Information Technology - Visual Informatics, ITSim'10, vol. 1, 2010. 